\title{
Sp(4, R) SYMMETRY IN LIGHT NUCLEI *
}

\author{
D. R. PETERSON ${ }^{\dagger}$ and K. T. HECHT ${ }^{+\dagger}$ \\ Physics Department, University of Michigan, Ann Arbor, Michigan 48109, USA
}

Received 26 March 1980

\begin{abstract}
A classification of nuclear states according to the non-compact Lie algebra $\operatorname{Sp}(4, R)$ is investigated. This model strikes a compromise between the $\operatorname{Sp}(6, R)$ and $S p(2, R)$ models and furnishes a practical, yet algebraically simple means for selecting those shell-model core excitations which are needed for the development of quadrupole collectivity in rotational bands of deformed nuclei. Applications to rotational bands in ${ }^{24} \mathrm{Mg}$ and ${ }^{16} \mathrm{O}$, including shell-model excitations with excitation energies up to $10 \mathrm{~h} \omega$, show that the core excitations needed to fit observed E2 rates in these nuclei are too large to be treated by perturbation theory. Despite this, a definite symplectic band structure emerges. The nature of the core excitations is very simple, so that it may be feasible to incorporate such symplectic excitation structures into more detailed shell-model calculations.
\end{abstract}

\section{Introduction}

Although there exists a highly successful phenomenological picture of collective excitations in nucle ${ }^{1}$ ), a truly satisfactory microscopic description has not yet been obtained. The strong correlations needed to build up the collective features of nuclear spectra seem to defy a description in the framework of the nuclear shell model since they seem to imply the need for shell-model matrices of enormous dimensions. The recent work of Rosensteel and Rowe ${ }^{2,3}$ ), however, shows that the non-compact algebra $S p(6, R)$ furnishes a natural algebraic tool for incorporating the core excitations associated with the nuclear quadrupole degree of freedom into the shell-model picture. Unlike earlier algebraic models of nuclear collective motion ${ }^{4-6}$ ), the symplectic Lie algebra $S p(6, R)$ contains the U(3) algebra of the harmonic oscillator shell model as a subalgebra. It is thus the natural generalization of the Elliott SU(3) model ${ }^{7}$ ) to include the effects of core excitations. $\operatorname{Sp}(6, R)$ symmetry provides a practical means for selecting those shell-model core excitations which are needed for the development of nuclear rotational spectra and may be a particularly powerful tool in those nuclei in the $A=8-28$ mass range in which conventional shell-model calculations indicate a high degree of SU(3) symmetry.

Although the $\operatorname{Sp}(6, \mathrm{R})$ algebra has long been known as the spectrum generating algebra for the 3-dimensional harmonic oscillator ${ }^{8}$ ), earlier applications of this algebra have led either to highly schematic models ${ }^{9}$ ) or have been used to derive

* Work supported by the US National Science Foundation.

Present address: Bell Laboratories, Holmdel, NJ 07733.

t† On leave of absence at Max-Planck-Institut für Kernphysik, D-6900 Heidelberg, W. Germany. 
results for harmonic oscillator functions in a single 3-dimensional variable ${ }^{10}$ ). The important implications of $\mathrm{Sp}(6, \mathrm{R})$ symmetry for nuclear many-body systems have been realized only recently by Rosensteel and Rowe ${ }^{2,3}$ ); [these authors use the notation $S p(3, R)]$. Independently, Arickx $\left.{ }^{11}\right)$ showed that the subalgebra $S p(2, R)$ led to a classification of states with a high degree of overlap with an earlier configuration interaction calculation ${ }^{12}$ ) for ${ }^{8} \mathrm{Be}$. This calculation attempted to extend the rotational band in ${ }^{8} \mathrm{Be}$ beyond the $\mathrm{SU}(3)$ limited maximum $L$-value and to build up the quadrupole collectivity of this band by the inclusion of core excitations. In this calculation the shell-model basis was restricted to $\mathrm{SU}(3)$ representations of maximum intrinsic deformation, with $(\lambda \mu)=(n+4,0)$, for core excitations with excitation energies $n \hbar \omega,(n=0,2,4)$. Since only oscillator excitations in 1 dimension, the direction of the intrinsic symmetry axis, were included, the subgroup $\operatorname{Sp}(2, \mathbf{R})$ was sufficient for the group theoretical interpretation of this new symmetry.

Matrix elements for the physically relevant discrete series of the non-compact group $\operatorname{Sp}(2, R)$ follow from simple generalizations of ordinary angular momentum theory $\left.{ }^{13-15}\right)$. Matrix elements for the generators of the non-compact $\operatorname{Sp}(6, R)$ algebra, in a basis symmetry-adapted to the U(3) subalgebra, are complicated by the problem of missing quantum numbers. The resultant inner multiplicity problem makes it impossible to give closed algebraic expressions for these matrix elements. A recursive procedure for the calculation of these matrix elements has recently been given by Rosensteel ${ }^{16}$ ). It involves an orthogonalization procedure for states with inner multiplicity [multiple occurences of $U(3)$ irreducible representations in a given symplectic irrep]. The computational difficulties associated with this recursive calculation are sufficient that detailed applications of the symplectic shell model of collective motion are only just beginning to be realized for very simple nuclear systems ${ }^{17}$ ).

The physics of the $S p(6, R) \supset U(3)$ shell model of collective excitations suggests a possible simplification. The most important SU(3) core excitations in a given symplectic band, [ $\infty$-dimensional irrep of $\mathrm{Sp}(6, \mathrm{R})$ ], may be those corresponding to excitations of largest intrinsic deformation, with large values of the Elliott quantum numbers $\lambda$ and $\mu$. The SU(3) representations of these excitations correspond to Young tableaux in which the added squares, induced by the symplectic excitation operators, increment only the 1 st and 2 nd rows of the tableaux. These oscillator excitations in 2 dimensions lead to $S U(3)$ irreps which can be generated by the $S p(4, R)$ subalgebra of the full $S p(6, R)$ algebra. The group chain $S_{p}(4, R) \supset S p(2, R) \times S p(2, R)$ leads to a complete set of quantum numbers for this symmetry and makes possible the use of simple and elegant algebraic techniques for constructing the irreducible representations. Neither SU(3) nor the ordinary 3-dimensional angular momentum subgroup are contained in the $\mathrm{Sp}(4, \mathrm{R})$ group. However, a simple algorithm can be set up for the construction of states of good SU(3) symmetry in the cartesian oscillator basis in terms of the eigenvectors of $\mathrm{Sp}(4, \mathrm{R}) \supset \mathrm{Sp}(2, \mathrm{R}) \times \mathrm{Sp}(2, \mathrm{R})$ symmetry. From these, SU(3) reduced matrix elements can then be combined with readily available 
$\mathrm{SU}(3) \supset 0_{3}^{+}$Wigner coefficients ${ }^{18}$ ) to construct the matrix elements of the $\mathrm{Sp}(6, \mathrm{R})$ generators in a basis with good SU(3) symmetry and orbital angular momentum.

It is the aim of this work to investigate the validity of this $\operatorname{Sp}(4, R)$ model. For this purpose an attempt is made to account for the experimentally observed E2 transition strengths in the rotational bands of deformed light nuclei without resorting to effective charges. In the conventional shell-model treatment of nuclear spectra the effect of excitations out of the closed-shell core and out of the valence shell into empty shells is treated by a renormalization of the residual interaction and other observables. These are replaced by effective operators acting in the space of the valence shells only ${ }^{19}$ ). The convergence difficulties associated with a perturbative treatment of this renormalization procedure are particularly marked in nuclei in which strongly deformed core-excited states sink down in energy to intrude into the region of normal shell-model states. The symplectic shell model of rotational bands is not dependent on such a perturbation approach. By explicitly building the quadrupole core excitations into the shell-model spacc, the symplectic model can account for the observed E2 transition rates without a renormalization of the E2 operator, that is without the introduction of effective (renormalized) charges. It is the aim of this work to investigate the nature and extent of the core excitations which are required to account for the observed quadrupole collectivity in light nuclei.

In sect. 2 a brief review is given of the $\operatorname{Sp}(6, R)$ algebra to establish notation and phase conventions; and the restricted classification according to its $\operatorname{Sp}(4, R)$ subalgebra is motivated further. The $\operatorname{Sp}(4, R)$ classification is developed in detail in sect. 3 , in which algebraic expressions are given for the matrix elements of the $\operatorname{Sp}(4 . R)$ generators. The symplectic shell model based on this subalgebra is described in sect. 4 , in which matrix elements of the quadrupole operator and a model hamiltonian are derived in a basis symmetry adapted to the desired $\mathrm{SU}(3) \supset 0_{3}^{+}$subgroup chain. Sect. 5 gives detailed applications to rotational bands with large observed E2 rates in the $S=0, T=0$ nuclei ${ }^{16} \mathrm{O},{ }^{20} \mathrm{Ne}$, and ${ }^{24} \mathrm{Mg}$.

\section{The $\operatorname{Sp}(6, R)$ model}

The symplectic shell model as formulated by Rosensteel and Rowe ${ }^{2.3}$ ) is generated by the $\mathrm{Sp}(6, \mathrm{R})$ Lie algebra which is formed by the 21 one-body operators

$$
\begin{gathered}
\tilde{A}_{i j} \equiv \frac{1}{2} \sum_{\alpha}^{A}\left\{a_{i}^{\dagger}(\alpha) a_{j}(\alpha)+a_{j}(\alpha) a_{i}^{\dagger}(\alpha)\right\}_{j}, \\
\tilde{B}_{i j}^{\dagger} \equiv \sum_{\alpha}^{A}\left\{a_{i}^{\dagger}(\alpha) a_{j}^{\dagger}(\alpha)\right\}=\tilde{B}_{j i}^{\dagger}, \\
\widetilde{B}_{i j} \equiv \sum_{\alpha}^{A}\left\{a_{i}(\alpha) a_{j}(\alpha)\right\} \equiv \widetilde{B}_{j i},
\end{gathered}
$$


given in terms of harmonic oscillator creation and annihilation operators; $(h \equiv 1)$

$$
\begin{gathered}
a_{i}^{\dagger}(\alpha)=\sqrt{\frac{1}{2} m \omega}\left(x_{i}(\alpha)-i / m \omega p_{i}(\alpha)\right), \\
a_{i}(\alpha)=\left(a_{i}^{\dagger}(\alpha)\right)^{\dagger},
\end{gathered}
$$

with $i, j=x, y, z ; \varkappa=$ particle index.

The $21 \mathrm{Sp}(6, \mathrm{R})$ generators can also be expressed in terms of the operators

$$
\begin{gathered}
L_{i j}=\sum_{\alpha}^{A}\left\{x_{i}(\alpha) p_{j}(\alpha)-x_{j}(\alpha) p_{i}(\alpha)\right\}, \\
Q_{i j}=\sum_{\alpha}^{A}\left\{x_{i}(\alpha) x_{j}(\alpha)\right\}, \\
K_{i j}=\sum_{\alpha}^{A}\left\{p_{i}(\alpha) p_{j}(\alpha)\right\}, \\
S_{i j}=\sum_{\alpha}^{A}\left\{x_{i}(\alpha) p_{j}(\alpha)+p_{i}(\alpha) x_{j}(\alpha)\right\} .
\end{gathered}
$$

Here, $L_{k}=\frac{1}{2} \varepsilon_{i j k} L_{i j}$ is the total orbital angular momentum operator of the $A$-particle system. The 6 operators $Q_{i j}$ can be decomposed into an $L-0$ tensor (monopole mass tensor) and a spherical tensor of rank $L=2$, the mass quadrupole operator. This is the real quadrupole operator, in contrast to the Elliott quadrupole operator

$$
\widetilde{Q}_{\text {Elliot }}=Q_{i j}+K_{i j}
$$

which is diagonal in the major oscillator quantum number and can not induce core excitations. The six $\widetilde{Q}_{i j}$ (Elliott), together with the three $L_{i j}$, constitute the nine operators $\tilde{A}_{i j}$ which generate the subgroup U(3). The operators $S_{i j}$ are the so-called shear stress tensors which generate the monopole and quadrupole deformations.

Contamination with spurious center of mass excitations can be eliminated [see ref. $\left.{ }^{11}\right)$ ] by replacing the oscillator creation and annihilation operators in the defining eqs. (1) by

$$
a_{i}^{\dagger}(\alpha) \rightarrow a_{i}^{\dagger}(\alpha)-\frac{1}{A} \sum_{\beta} a_{i}^{\dagger}(\beta) .
$$

The desired realization of $\operatorname{Sp}(6, \mathrm{R})$ is then

$$
\begin{gathered}
A_{i j}=\tilde{A}_{i j}-\frac{1}{2} A \sum_{\alpha, \beta}^{A}\left\{a_{i}^{\dagger}(\alpha) a_{j}(\beta)+a_{j}(\beta) a_{i}^{\dagger}(\alpha)_{j}^{\prime},\right. \\
B_{i j}^{\dagger}=\tilde{B}_{i j}^{\dagger}-\frac{1}{A} \sum_{\alpha, \beta}^{A}\left\{a_{i}^{\dagger}(\alpha) a_{j}^{\dagger}(\beta)\right\}=B_{j i}^{\dagger}, \\
B_{i j}=\widetilde{B}_{i j}-\frac{1}{A} \sum_{\alpha, \beta}^{A}\left\{a_{i}(\alpha) a_{j}(\beta)\right\}=B_{j i} .
\end{gathered}
$$


The operators $A_{i j}, B_{i j}^{\dagger}, B_{i j}$ are mixed one- and two-body operators. However, they satisfy the same commutator algebra as the one-body operators $\tilde{A}_{i j}, \tilde{B}_{i j}^{\dagger}, \widetilde{B}_{i j}$ of eq. (1).

Even though the nuclear states possess good angular momentum, it is more convenient to construct the representations of $S p(6, R)$ by classifying states via the chain

$$
\mathrm{Sp}(6, \mathrm{R}) \supset \mathrm{U}(3) \supset \mathrm{SU}(3) \supset \mathrm{SU}(2) \times \mathrm{U}(1) .
$$

$\mathrm{SU}(3)$ reduced matrix elements are calculated more easily in this chain. These reduced matrix elelements can then be combined with $\mathrm{SU}(3) \supset 0_{3}^{+}$Wigner coefficients to regain good angular momentum via the Wigner-Eckart theorem. The oscillator creation operators have the following $\mathrm{SU}(3) \supset \mathrm{U}(1) \times \mathrm{SU}(2)$ irreducible tensor character

$$
a_{z}^{\dagger}=T_{200}^{(10)}, \quad a_{x}^{\dagger}=T_{-1 \frac{1}{2} \frac{1}{2}}^{(10)}, \quad a_{y}^{\dagger}=T_{-1 \frac{1}{2}-\frac{1}{2}}^{(10)},
$$

where $T_{\varepsilon A M}^{(\lambda \mu)}$ is a tensor operator transforming according to $(\lambda \mu)$ under $\operatorname{SU}(3)$, while $\varepsilon \tilde{\Lambda} M_{\lambda}$ are the Elliott $\mathrm{U}(1) \times \mathrm{SU}(2)$ subgroup labels ${ }^{7}$ ). (A tilde is used for the Elliott quantum numbers $\tilde{\Lambda}$ to distinguish these from the bare $A$ which will have a different significance in sect. 3 .) The phase convention under hermitian conjugation is

$$
\left(T_{\varepsilon \tilde{X} M \tilde{A}}^{(\lambda \mu)}\right)^{\dagger}=(-1)^{1 / 3(\lambda-\mu)+M \tilde{A}-\varepsilon / 6} T_{-\varepsilon \tilde{\lambda}-M \tilde{A}}^{(\lambda \mu)}
$$

By coupling the fundamental tensors, (7), with their hermitian conjugates, the $\operatorname{Sp}(6, R)$ generators can be expressed in terms of definite SU(3) irreducible tensor character

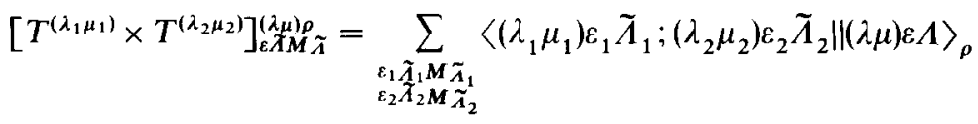

$$
\begin{aligned}
& \times\left\langle\tilde{\Lambda}_{1} M_{\tilde{A}_{1}} \tilde{\Lambda}_{2} M_{\tilde{A}_{2}} \mid \tilde{\Lambda} M_{\tilde{A}}\right\rangle T_{\varepsilon_{1} \tilde{X}_{1} M_{\tilde{A}_{1}}}^{\left(\lambda_{1} \mu_{1}\right)} T_{\varepsilon_{2} \tilde{X}_{2} M_{\tilde{A}_{2}}}^{\left(\lambda_{2} \mu_{2}\right)} .
\end{aligned}
$$

Here $\left\langle\left(\lambda_{1} \mu_{1}\right) \varepsilon_{1} \tilde{\Lambda}_{1} ;\left(\lambda_{2} \mu_{2}\right) \varepsilon_{2} \tilde{\Lambda}_{2} \|(\lambda \mu) \varepsilon \tilde{\Lambda}\right\rangle_{\rho}$ is a reduced SU(3) Wigner coefficient, where $\rho$ labels the outer multiplicity for the coupling $\left(\lambda_{1} \mu_{1}\right) \times\left(\lambda_{2} \mu_{2}\right) \rightarrow(\lambda \mu)$. The $\mathrm{SU}(3) \supset \mathrm{U}(1) \times \mathrm{SU}(2)$ irreducible tensor character of the $\mathrm{Sp}(6, \mathrm{R})$ generators is shown explicitly in table 1 . The $\mathrm{Sp}(6, \mathrm{R})$ generators can also be subdivided into nine Cartan-Weyl raising operators

$$
T^{(20)}, \quad A_{x z}, \quad A_{y z}, \quad A_{y x},
$$

nine lowering operators

$$
T^{(02)}, \quad A_{z x}, \quad A_{z y}, \quad A_{x y},
$$

and the three operators

$$
H_{i} \equiv A_{i i}=\sum_{\alpha}^{A} a_{i}^{\dagger}(\alpha) a_{i}(\alpha)+\frac{1}{2}(A-1)-\frac{1}{A}\left(\sum_{\alpha}^{A} a_{i}^{\dagger}(\alpha)\right)\left(\sum_{\beta}^{A} a_{i}(\beta)\right),
$$

which determine the weights of the $\operatorname{Sp}(6, R)$ representation. Every $S U(3)$ highest weight state for a closed shell-model core + valence shell configuration, (with 0 h $\omega$ 
TABLE 1 A

$\mathrm{SU}(3) \supset \mathrm{SU}(2) \times \mathrm{U}(1)$ tensor character of $\mathrm{U}(3)$ subalgebra

$$
\begin{aligned}
& (\lambda \mu)=(00) \\
& T^{(00)}=\sqrt{\frac{\pi}{3}}\left\{A_{x x}+A_{y x}+A_{z z}\right\} \\
& (\lambda \mu)=(11) \\
& T_{31 / 21 / 2}^{(11)}=-\sqrt{\frac{T}{2}} A_{z y} \quad T_{-31 / 2-1 / 2}^{(11)}=-\sqrt{\frac{T}{2}} A_{y z} \\
& T_{31 / 2-1 / 2}^{(11)}=\sqrt{\frac{1}{2}} A_{z x} \quad T_{-31 / 21 / 2}^{(11)}=-\sqrt{\frac{1}{2}} A_{x z} \\
& T_{011}^{(11)}=-\sqrt{\frac{1}{2}} A_{x y} \equiv-\sqrt{\frac{1}{2}} \tilde{A}_{+} \\
& T_{010}^{(11)}=\frac{1}{2}\left(A_{x x}-A_{y y}\right) \equiv \tilde{\lambda}_{0} \\
& T_{01-1}^{1111}=\sqrt{\frac{1}{2}} A_{y x} \equiv \sqrt{\frac{1}{2}} \tilde{\Lambda}_{-} \\
& T_{000}^{(11)}=-\frac{1}{2} \sqrt{\frac{1}{3}}\left(2 A_{z z}-A_{x x}-A_{y y}\right) \equiv-\frac{1}{2} \sqrt{\frac{1}{3}} \tilde{Q}_{0}
\end{aligned}
$$

\begin{tabular}{|c|c|}
\hline$(\lambda \mu)=(20)$ & $(\lambda \mu)=(02)$ \\
\hline $\begin{aligned} T_{400}^{(20)} & =\sqrt{\frac{1}{2}} B_{z z}^{\dagger} \\
T_{1}^{(20)}{ }_{1 / 21 / 2}^{\prime} & =B_{z x}^{\dagger}\end{aligned}$ & $\begin{aligned} T_{-400}^{1021} & =\sqrt{\frac{1}{2}} B_{z z} \\
T_{-1}^{(02)} & =-B_{z x}-1 / 2=\end{aligned}$ \\
\hline$T_{11 / 2-1 / 2}^{(2 n)}=B_{z y}^{+}$ & $T_{-11 / 21 / 2}^{(02)}=B_{z y}$ \\
\hline$T_{-211}^{(20)}=\sqrt{\frac{1}{2}} B_{x x}^{\dagger}$ & $T_{21-1}^{(02)}=\sqrt{\frac{1}{2}} B_{x x}$ \\
\hline$T_{-210}^{(0)}=B_{x y}^{+}$ & $T_{210}^{\mathrm{n} \geqslant \mathrm{n}}=-\boldsymbol{B}_{x y}$ \\
\hline$T_{-21-1}^{(20)}=\sqrt{\frac{T}{2}} B_{y y}^{\dagger}$ & $T_{211}^{(02)}=\sqrt{\frac{T}{2}} B_{y y}$ \\
\hline
\end{tabular}

\section{TABLE 1B}

$\mathrm{SU}(3)=\mathrm{SU}(2) \times \mathrm{U}(1)$ tensor character of $B_{i j}^{\dagger}$ and $B_{i j}$

excitation energy) is automatically annihilated by the nine lowering operators. Such a state can be described by a 3-rowed tableau with $\lambda_{0}+\mu_{0}+v_{0}$ squares in the 1st row (maximum possible number of $z$ quanta), $\mu_{0}+v_{0}$ squares in the 2nd row (maximum number of $x$ quanta in the state with a maximum $n_{z}$ ), and $v_{0}$ squares in the 3 rd row, and will be denoted by

$$
\left|\left\{\lambda_{0} \mu_{0} v_{0}\right\}\left(\lambda_{0} \mu_{0}\right) v_{0} \mathrm{HW}\right\rangle .
$$

It forms the band head for the $\infty$-dimensional $\operatorname{Sp}(6, \mathbf{R})$ representations, (discrete positive series). It is more convenient to use the simple labels $\lambda_{0}, \mu_{0}$ and $v_{0}$ in place of the band-head eigenvalues of $H_{1}, H_{2}$ and $H_{3}$. The raising operators generate the states

$$
\left|\left\{\lambda_{0} \mu_{0} v_{0}\right\}(\lambda \mu) v \varepsilon, \tilde{\Lambda} M_{X} \gamma\right\rangle,
$$

with $\mathrm{SU}(3)$ quantum numbers $(\lambda \mu)$, carrying $\lambda+2 \mu+3 v=\lambda_{0}+2 \mu_{0}+3 v_{0}+2 N$ 
oscillator quanta, $(N=$ integer $)$, Elliott $\mathrm{U}(1) \times \mathrm{SU}(2)$ subgroup labels $\varepsilon \widetilde{\lambda} M_{\tilde{A}}$, and an additional label $\gamma$ to distinguish states with the same $(\lambda \mu)$ in the case of multiple occurences. [ $\gamma$ carries the burden of the 3 missing quantum numbers in the $\mathrm{Sp}(6, R) \supset \mathrm{U}(3) \supset \mathrm{U}(1) \times \mathrm{SU}(2)$ group chain). Such a state can be generated by the action of $N$ raising operators $T^{(20)}$

$\left|\left\{\lambda_{0} \mu_{0} v_{0}\right\}(\lambda \mu) v \varepsilon \tilde{\Lambda} M_{\lambda} \gamma\right\rangle$

$$
=\left[\left[T^{(20)} \times \ldots \times T^{(20)}\right]^{(\bar{\lambda} \bar{\mu})} \times\left|\left\{\lambda_{0} \mu_{0} v_{0}\right\}\left(\lambda_{0} \mu_{0}\right) \nu_{0}\right\rangle\right]_{\varepsilon \tilde{B}}^{(\lambda \mu \mu)}
$$

where the square bracket denotes $\mathrm{SU}(3)$ coupling, and where the possible $(\bar{\lambda} \bar{\mu})$ follow from the simple rule ${ }^{20}$ ): Construct all possible 3-rowed tableaux consisting of $N$ squares with $f_{i}=$ number of squares in the $i$ th row, $\sum_{i} f_{i}=N$. Then

$$
(\bar{\lambda} \bar{\mu})=\left(2 f_{1}-2 f_{2}, 2 f_{2}-2 f_{3}\right) \text {. }
$$

The spectrum of $S U(3)$ irreps for a given symplectic band is then given by

$$
\{(\lambda \mu)\}=\sum_{(\lambda \mu)}\left\{(\overline{\lambda \mu}) \otimes\left(\dot{\lambda}_{0} \mu_{0}\right)\right\} .
$$

In general, a raising operator, $T^{(20)}$, when acting on a state of definite $(\lambda \mu) v$ will lead to a linear combination of six states $\left(\lambda^{\prime} \mu^{\prime}\right) v^{\prime}$ with $\lambda^{\prime}+2 \mu^{\prime}+3 v^{\prime}=\lambda+2 \mu+3 v+2$. Note, however, that the operator, $T_{400}^{(20)}$, ladders between SU(3) highest weight (HW) states only:

$$
T_{400}^{(20)}|(\lambda \mu) \mathrm{HWW}\rangle=\mathrm{const}|(i+2, \mu) v \mathrm{HW}\rangle .
$$

It is desirable to construct six independent operators from the $\operatorname{Sp}(6, R)$ algebra which have this simple property. Such operators, $O_{i j}^{\dagger}$, follow from the three equations

$$
\left.\left\{A_{z y} O_{i j}^{\dagger}=A_{z x} O_{i j}^{\dagger}=A_{x y} O_{i j}^{\dagger}\right\}(\lambda \mu) \mu \mathrm{HW}\right\rangle=0 .
$$

For example,

$$
\begin{gathered}
O_{z z}^{\dagger}=\frac{1}{2} B_{z z}^{\dagger}, \\
O_{z x}^{\dagger}=B_{z x}^{\dagger}\left(H_{1}-H_{2}+2\right)-2 A_{x z} O_{z z}^{\dagger}, \\
O_{x x}^{\dagger}=\frac{1}{2} B_{x x}^{\dagger}\left(H_{1} H_{2}+1\right)\left(H_{1}-H_{2}\right)-A_{x z} O_{z x}^{\dagger}\left(H_{1}-H_{2}+1\right)\left(H_{1}-H_{2}+2\right)^{-1} \\
-A_{x z} A_{x z} O_{z z}^{\dagger}\left(H_{1}-H_{2}\right)\left(H_{1}-H_{2}+2\right)^{-1},
\end{gathered}
$$

where these ladder between states $|(\lambda \mu) \mathrm{HW}\rangle$ and $\left|\left(\lambda^{\prime} \mu^{\prime}\right) \mathrm{HW}\right\rangle$ with $\left(\lambda^{\prime} \mu^{\prime}\right)=(\lambda+2, \mu)$, $(\lambda, \mu+1)$, and $(\lambda-2, \mu+2)$, respectively. (For normalization factors, and for the more complicated operators, $O_{i j}^{\dagger}$, which add quanta in the $y$-direction, see ref. ${ }^{21}$ ). Although the complete set of states for a symplectic band can, in principle, be constructed by successive application of these operators; in practice this constructive process is complicated by the multiplicity problem. E.g., there are two independent states with 
$(\lambda \mu)=\left(\lambda_{0}, \mu_{0}+2\right) \gamma_{0}$ in the $\operatorname{Sp}(6, \mathrm{R})$ band $\left\{\hat{\lambda}_{0} \mu_{0} v_{0}\right\}$. Two independent states of this type can be constructed by the action of the operators $O_{z z}^{\dagger} O_{x x}^{\dagger}$ and $O_{z x}^{\dagger} O_{z x}^{\dagger}$; but these states are not orthogonal. Although a Grahm-Schmidt procedure can be used to construct an orthonormal basis, the combination of laddering and orthogonalization steps becomes more and more complicated for higher excitations and is not amenable to a solution in closed algebraic form.

The physics of quadrupole collectivity suggests a possible simplification. The most important $S U(3)$ core excitations in a given $S p(6, R)$ band are those with largest intrinsic deformation, that is those with large values of $\lambda$ and $\mu$. States which are energetically favored by an attractive quadrupole-quadrupole interaction are those characterized by a large eigenvalue of the $S U(3)$ Casimir invariant

$$
\tilde{C}_{\mathrm{SU}(3)}=\frac{1}{9}\left\{\hat{\lambda}^{2}+\mu^{2}+\hat{\lambda} \mu+3 \hat{\lambda}+3 \mu\right\} .
$$

The effect of an $O_{i j}^{\dagger}$ on $\tilde{C}_{\mathrm{SU}(3)}$ is given by $\Delta \tilde{C}_{i j}=\widetilde{C}_{\mathrm{SU}(3)}\left(\lambda^{\prime} \mu^{\prime}\right)-\widetilde{C}_{\mathrm{SU}(3)}(\lambda \mu)$. E.g.,

$$
\begin{gathered}
\Delta \tilde{C}_{z z}=+\frac{2}{9}\{2 \lambda+\mu+5\}, \\
\Delta \tilde{C}_{x x}=+\frac{2}{9}\{\mu-\lambda+2\}, \quad \Delta \tilde{C}_{y y}=-\frac{2}{9}\{2 \mu+\lambda+1\} .
\end{gathered}
$$

There is thus a hierarchy for determining the most important states for a structure calculation. This can be summarized symbolically by

$$
O_{y y}^{\dagger}<O_{x y}^{\dagger}<O_{z y}^{\dagger}<O_{x x}^{\dagger}<O_{z x}^{\dagger}<O_{z z}^{\dagger} \text {. }
$$

The most important core excitations are thus generated by the operators $O_{z z}^{\dagger}, O_{z x}^{\dagger}$ and $O_{x x}^{\dagger}$, which are constructed from operators of the subalgebra $\operatorname{Sp}(4, \mathrm{R}) ;\left(B_{i j}^{\dagger}\right.$, $B_{i j}$ and $A_{i j}$ with $\left.i, j=z, x\right)$. The success of the $\mathrm{Sp}(2, \mathrm{R})$ model of collective motion ${ }^{11,22}$ ) as applied to ${ }^{8} \mathrm{Be}$, with its large prolate intrinsic deformation, lends further support to a truncation employing the $\operatorname{Sp}(4, R)$ algebra. The $S p(4, R)$ model strikes a compromise between the two extreme models, $\operatorname{Sp}(2, R)$ and $\operatorname{Sp}(6, R)$, and avoids most of the computational complexities associated with the full $\mathrm{Sp}(6, \mathrm{R})$ model. By excluding excitations in the intrinsic $y$-direction from the model space, but allowing excitations in both $x$-and $z$-directions, core excitations with large values of both SU(3) quantum numbers $\lambda$ and $\mu$ are included in the model space. This may be particularly important in nuclei such as ${ }^{24} \mathrm{Mg}$ with relatively large values for both $\lambda_{0}$ and $\mu_{0}$, and in nuclei such as ${ }^{28} \mathrm{Si}$ where there may be competition between prolate deformations (large $\lambda$, small $\mu$ ) and oblate deformations (small $\lambda$, large $\mu$ ).

\section{The $S p(4, R)$ classification}

Since the group chain $S p(4, R) \supset S p(2, R) \times S p(2, R)$ leads to a complete set of commuting operators, it is possible to construct states of the physically relevant discrete positive series of this noncompact group by algebraic techniques. The SU(3) irreps contained in this construction can be made manifest in this mathemati- 
cally natural basis for $\operatorname{Sp}(4, \mathrm{R})$, so that a simple algorithm can be constructed for projecting out states of good $S U(3) \supset S U(2) \times U(1)$ symmetry. From the latter it is then straightforward to evaluate the SU(3) reduced matrix elements needed for applications of the $S p(4, R)$ model of collective excitations.

By restricting the oscillator excitations to the $z$ - and $x$-directions the following non-compact $\operatorname{Sp}(4, \mathrm{R})$ Lie algebra is obtained ${ }^{23}$ ). (The symbols $A, M_{A}$ should not be confused with the $\mathrm{SU}(3)$ subgroup labels $\tilde{A}, M_{\tilde{A}}$ introduced in sect. 2.)

$\operatorname{Sp}(2, R)_{z}$ :

$$
\Lambda_{+}=\frac{1}{2} \sqrt{ } \frac{1}{2} B_{z z}^{\dagger}, \quad A_{0}=\frac{1}{2} H_{1}, \quad \Lambda_{-}=-\frac{1}{2} \sqrt{ } \frac{1}{2} B_{z z} .
$$

$\operatorname{Sp}(2, R)_{x}:$

$$
\Sigma_{+}=\frac{1}{2} \sqrt{ } \frac{1}{2} B_{x x}^{\dagger}, \quad \Sigma_{0}=\frac{1}{2} H_{2}, \quad \Sigma_{-}=-\frac{1}{2} \sqrt{ } \frac{1}{2} B_{x x} .
$$

$T_{M M_{\Sigma}}^{A \Sigma}:$

$$
\begin{aligned}
B_{z x}^{\dagger} & =T_{\frac{1}{2} \frac{1}{2} \frac{1}{2}}^{\frac{1}{2}}, \quad B_{z x}=T_{-\frac{1}{2}-\frac{1}{2}}^{\frac{1}{2}}, \\
-A_{z x} & =T_{\frac{1}{2}-\frac{1}{2}}^{\frac{1}{2}}, \quad-A_{x z}=T_{-\frac{1}{2} \frac{1}{2} \frac{1}{2}} .
\end{aligned}
$$

The operators $\Lambda_{+}, A_{-}, \Lambda_{0}$ generate the $\mathrm{Sp}(2, \mathrm{R})$ subalgebra. Note that the phases are chosen so that these satisfy the usual angular momentum commutation relations (including signs). Therefore $\Lambda_{+}$and $\Lambda_{-}$are $M_{A}$ raising and lowering operators for states with eigenvalues $M_{A}$ for $\Lambda_{0}$. However, $A_{+}^{\dagger}=-A_{-}$. The extra minus sign changes the form of the Casimir operator, when expressed in terms of the three hermitian operators $\Lambda_{x}, \Lambda_{y}, \Lambda_{z}$, with $\Lambda_{+}=\sqrt{ } \frac{1}{2}\left(\Lambda_{x}+i \Lambda_{y}\right)$.

$$
C_{A}^{(2)}=\Lambda_{0}^{2}-\Lambda_{0}+2 A_{+} A_{-}=\Lambda_{z}^{2}-\left(\Lambda_{x}^{2}+\Lambda_{y}^{2}\right) \text {. }
$$

As a result ${ }^{13-15}$ ), (i) the $M_{A}$ ladders are $\infty$-dimensional, (ii) there is a lowest $M_{A}$ value, $A$, for which $\Lambda_{-}\left|C_{A} M_{A}=\Lambda\right\rangle=0$, (iii) $C_{A}^{(2)}$ has the eigenvalue $C_{A}=A(\Lambda-1)$ and (iv) $M_{A}=A, A+1, \Lambda+2, \ldots$ for the discrete + series, (the only series needed for the harmonic oscillator excitation problem). The operators $\Sigma_{+}, \Sigma_{-}, \Sigma_{0}$ generate $\operatorname{Sp}(2, \mathbf{R})_{x}$ which commutes with $\operatorname{Sp}(2, \mathbf{R})_{z}$ and leads to the discrete + series with $M_{\Sigma}=\Sigma, \Sigma+1, \Sigma+2, \ldots$, with $C_{\Sigma}=\Sigma(\Sigma-1)$. The four operators $A_{0}, C_{A}^{(2)}, \Sigma_{0}, C_{\Sigma}^{(2)}$ form a complete set of commuting operators for the subgroup lables of the 10 parameter, rank-2 $\operatorname{Sp}(4, \mathrm{R})$ algebra. The 2 quantum numbers needed to label the unitary irreps of $\operatorname{Sp}(4, \mathbf{R})$ are taken to be the eigenvalues of $\Lambda_{0}$ and $\Sigma_{0}$ when acting on a $\operatorname{Sp}(4, R)$ lowest weight state. This state, to be denoted by $|0\rangle$, is just the $\operatorname{Sp}(6, R)$ lowest weight state, the symplectic band head $\left|\left\{\lambda_{0} \mu_{0} v_{0}\right\}\left(\lambda_{0} \mu_{0}\right) v_{0} \mathrm{HW}\right\rangle$ introduced in sect. 2. Noting that $A_{0}=\frac{1}{2} H_{1}=\frac{1}{2} A_{z z}, \Sigma_{0}=\frac{1}{2} H_{2}=\frac{1}{2} A_{x x}$. [see eq. (10c)],

$$
\begin{gathered}
\Lambda_{0}|0\rangle=\bar{\Lambda}|0\rangle, \quad \bar{\Lambda}=\frac{1}{2}\left\{\lambda_{0}+\mu_{0}+v_{0}+\frac{1}{2}(A-1)\right\}, \\
\Sigma_{0}|0\rangle=\bar{\Sigma}|0\rangle, \quad \bar{\Sigma}=\frac{1}{2}\left\{\mu_{0}+v_{0}+\frac{1}{2}(A-1)\right\}, \\
\left\{\Lambda_{-}=\Sigma_{-}=A_{z x}=B_{z x}\right\}|0\rangle=0 .
\end{gathered}
$$


The discrete series $D^{++}(\bar{\Lambda}, \Sigma)$ can thus be characterized by the quantum numbers $\bar{\Lambda}, \bar{\Sigma}$, and the basis vectors can be denoted by $\left|\bar{\Lambda} \bar{\Sigma} \Lambda M_{\Lambda} \Sigma M_{\Sigma}\right\rangle$. These are simultaneous eigenstates of $\Lambda_{0}, C_{A}^{(2)}, \Sigma_{0}$ and $C_{\Sigma}^{(2)}$ and thus form an orthogonal basis. The $\operatorname{Sp}(4, \mathrm{R})$ lowest weight state $|0\rangle \equiv|\bar{\Lambda} \bar{\Sigma} \bar{\Lambda} \bar{\Lambda} \bar{\Sigma} \bar{\Sigma}\rangle$. Note that $\bar{\Lambda}, \bar{\Sigma}$ will be $\frac{1}{4}$ integral for even nuclei. [These are not faithful irreps of $S p(4, R)$, see refs. ${ }^{8,13}$ ). Since finite transformations of $S p(4, R)$ play no role in what follows, it will be sufficient to construct the eigenstates of the non-compact Lie algebra $\mathrm{Sp}(4, \mathrm{R})$.] Starting with an $\operatorname{Sp}(2$, $\mathrm{R})_{z} \times \mathrm{Sp}(2, \mathrm{R})_{x}$ lowest weight state $\left(M_{\Lambda}=\Lambda, M_{z}=\Sigma\right), \Lambda_{+}$and $\Sigma_{+}$can be applied an arbitrary number of times to yield

$$
\left|\bar{\Lambda} \bar{\Sigma} \Lambda M_{A} \Sigma M_{\Sigma}\right\rangle=N\left(\Lambda_{+}\right)^{M_{\Lambda}-\Lambda}\left(\Sigma_{+}\right)^{M_{\Sigma}-\Sigma}|\bar{\Lambda} \bar{\Sigma} \Lambda \Lambda \Sigma \Sigma\rangle,
$$

where $N$ is a normalization factor. The four operators $B_{z x}^{\dagger}, B_{z x}, A_{z x}$ and $A_{x z}$ transform according to 2-dimensional (non-unitary) representations of the subgroups $\operatorname{Sp}(2, \mathrm{R})_{z}$ and $\operatorname{Sp}(2, R)_{x^{\prime}}$. They can thus be characterized as tensor operators $T_{M_{A}{ }^{\prime \prime} \Sigma_{\Sigma}^{\prime \prime}}^{\prime \prime}$, with $\Lambda^{\prime \prime}=\frac{1}{2}$ and $\Sigma^{\prime \prime}=\frac{1}{2}$. When acting on a state $\left|\bar{\Lambda} \bar{\Sigma} \Lambda M_{A} \Sigma M_{\Sigma}\right\rangle$ they will generatc a linear combination of four vectors $\left|\bar{\Lambda} \bar{\Sigma} \Lambda^{\prime} M_{\Lambda}^{\prime} \Sigma^{\prime} M_{\Sigma}^{\prime}\right\rangle$ with $\Lambda^{\prime}=\Lambda \pm \frac{1}{2}, \Sigma^{\prime}=\Sigma \pm \frac{1}{2}$ but with fixed $M_{A}^{\prime}=M_{A}+M_{A}^{\prime \prime}$ and $M_{\Sigma}^{\prime}=M_{\Sigma}+M_{\Sigma}^{\prime \prime}$. These operators can be used to construct $A$ and $\Sigma$ raising and lowering operators. The construction of the general state $\left|\bar{\Lambda} \bar{\Sigma} \Lambda M_{A} \Sigma M_{A}\right\rangle$ will proceed in two steps. The 1st step consists of constructing an $\operatorname{Sp}(2, \mathrm{R})_{z} \times \operatorname{Sp}(2, \mathrm{R})_{x}$ lowest weight state $|\bar{\Lambda} \bar{\Sigma} \Lambda \Lambda \Sigma \Sigma\rangle$, starting with $|\bar{\Lambda} \bar{\Sigma} \bar{\Lambda} \overline{\bar{\Sigma}} \bar{\Sigma}\rangle$. In the 2nd step states with $M_{A}>\Lambda, M_{\Sigma}>\Sigma$ are then constructed by the $M$-raising operators $\Lambda_{+}$and $\Sigma_{+}$, through eq. (23). The 1st step is accomplished by operators $O_{\alpha \beta}$ with the property

$$
O_{\alpha \beta}|\bar{\Lambda} \bar{\Sigma} \Lambda \Lambda \Sigma \Sigma\rangle=\text { const }|\bar{\Lambda} \bar{\Sigma} \Lambda+\alpha \Lambda+\alpha \Sigma+\beta \Sigma+\beta\rangle,
$$

where $\alpha, \beta= \pm \frac{1}{2}$. Such operators must obey the relations

$$
\begin{gathered}
{\left[\Lambda_{0}, O_{\alpha \beta}\right]=\alpha O_{\alpha \beta}, \quad\left[\Sigma_{0}, O_{\alpha \beta}\right]=\beta O_{\alpha \beta},} \\
\Lambda_{-} O_{\alpha \beta}|\bar{\Lambda} \bar{\Sigma} \Lambda \Lambda \Sigma \Sigma\rangle=\Sigma_{-} O_{\alpha \beta}|\bar{\Lambda} \bar{\Sigma} \Lambda \Lambda \Sigma \Sigma\rangle=0
\end{gathered}
$$

These relations lead to

$$
\begin{gathered}
O_{-+}=A_{x z}\left(\Sigma_{0}-\frac{1}{2}\right)-\sqrt{\frac{1}{2}} \Sigma_{+} B_{z x} \\
O_{+-}=A_{z x}\left(\Lambda_{0}-\frac{1}{2}\right)-\sqrt{\frac{1}{2}} \Lambda_{+} B_{z x}, \\
O_{++}=B_{2 x}^{\dagger}\left(\Lambda_{0}-\frac{1}{2}\right)\left(\Sigma_{0}-\frac{1}{2}\right)-\sqrt{\frac{1}{2}} \Lambda_{+} A_{x z}\left(\Sigma_{0}-\frac{1}{2}\right) \\
-\sqrt{\frac{1}{2}} \Sigma_{+} A_{z x}\left(\Lambda_{0}-\frac{1}{2}\right)+\frac{1}{2} \Lambda_{+} \Sigma_{+} B_{z x} \\
O_{--}=B_{z x^{\circ}}
\end{gathered}
$$

The possible $A, \Sigma$ values in the representation $\bar{\Lambda} \bar{\Sigma}$ are given by ${ }^{21,23}$ )

$$
\Lambda=\bar{\Lambda}+\frac{1}{2}(m-n), \quad \Sigma=\bar{\Sigma}+\frac{1}{2}(m+n),
$$




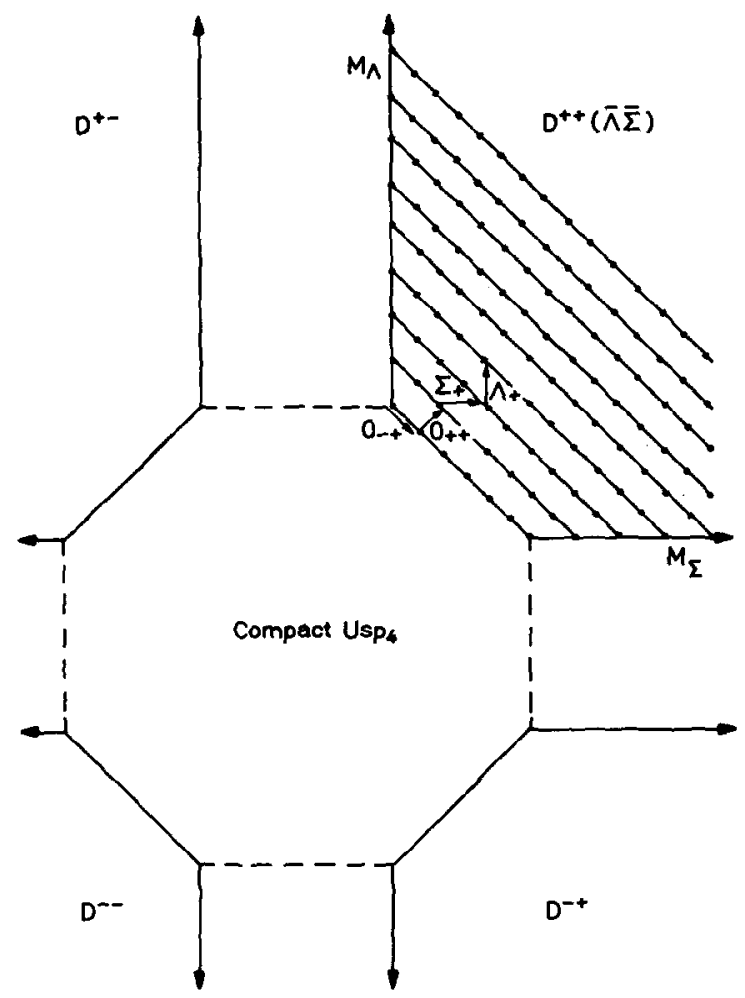

Fig. 1. Weight space, $\left(M_{A}, M_{\Sigma}\right)$, for various classes of irreps.

with

$$
m=0,1,2,3, \ldots, \quad 0 \leqq n \leqq 2(\bar{\Lambda}-\bar{\Sigma}) \equiv \hat{\lambda}_{0} .
$$

With $M_{A}=\Lambda+k, M_{\Sigma}=\Sigma+l ; k, l=0,1,2,3, \ldots$, the general state is given by

$$
\left.\left|\bar{\Lambda} \bar{\Sigma} \Lambda M_{A} \Sigma M_{\Sigma}\right\rangle \equiv|\bar{\Lambda} \bar{\Sigma} k| m n\right\rangle
$$

$$
=N(\bar{\Lambda}, \bar{\Sigma}, k, l, m, n)\left(A_{+}\right)^{k}\left(\Sigma_{+}\right)^{l}\left(O_{++}\right)^{m}\left(O_{-+}\right)^{n}|\bar{\Lambda} \bar{\Sigma} \bar{\Lambda} \bar{A} \bar{\Sigma} \bar{\Sigma}\rangle .
$$

In fig. 1 the action of the operators $\Lambda_{+}, \Sigma_{+}, O_{++}, O_{-+}$are indicated in the 2-dimensional weight space $M_{A}, M_{\Sigma}$ of a general irrep $D^{++}(\bar{\Lambda} \Sigma)$. Since $O_{++}$and $O_{-+}$ commute, when acting on a state with $M_{A}=A, M_{\Sigma}=\Sigma$, the order of $O_{++}, O_{-+}$ in eq. (28) is unimportant. Since $B_{z x}|0\rangle=0$, and $\left[B_{z x}, A_{x z}\right]=-2 \sqrt{2} A_{-},\left(O_{-+}\right)^{n}|0\rangle$ reduces to $\left(\bar{\Sigma}-\frac{1}{2}\right)^{n} A_{x z}|0\rangle$. The generator $A_{x z}$ is an $\mathrm{SU}(3)$ lowering operator and can be applied to $|0\rangle=\left|\left\{\lambda_{0} \mu_{0} v_{0}\right\}\left(\lambda_{0} \mu_{0}\right) v_{0} \mathrm{HW}\right\rangle$ at most $\lambda_{0}$ times $\left.{ }^{7,24}\right)$. Thus, $0 \leqq n \leqq \lambda_{0}$. However, the + integers $m, k, l$ can be arbitrarily large.

The norm factor $N(k, l, m, n)$ of eq. (28) is conveniently expressed in factored form

$$
N(k, l, m, n)=N(k, m, n) N(l, m, n) N(m, n),
$$


where the norm factor for states with $k=l=0$ has been evaluated by a recursive process ${ }^{21}$ )

$$
\begin{aligned}
N(m, n)=\left[\frac{2^{4 m+2 n}(2 \bar{A}-n-2) !(2 \bar{\Sigma}+n-2) !(2 \bar{A}+2 \bar{\Sigma}-3) !}{m ! n !(2 \bar{A}+m-n-2) !(2 \bar{\Sigma}+m+n-2) !(2 \bar{A}+2 \bar{\Sigma}+m-3) !}\right. \\
\left.\quad \times \frac{(2 \bar{A}-1) !(2 \bar{\Sigma}-2) !(2 \bar{A}-2 \bar{\Sigma}-n) ![(2 \bar{\Sigma}-2) !]^{2}}{(2 \bar{A}+m-1) !(2 \bar{\Sigma}+m-2) !(2 \bar{A}-2 \bar{\Sigma}) ![(2 \bar{\Sigma}+n-2) !]^{2}}\right]^{\frac{1}{2}},
\end{aligned}
$$

where $x ! \equiv \Gamma(x+1) / \Gamma\left(\frac{1}{2}\right)$ when $x=\frac{1}{2}$-integral. The $\mathrm{Sp}(2, \mathrm{R})$ norm factors are given by ${ }^{21}$ )

$$
\begin{array}{ll}
N(k, m, n)=\left[\frac{2^{k}(2 A-1) !}{k !(2 A+k-1) !}\right]^{\frac{1}{2}}, & \Lambda=\bar{A}+\frac{1}{2}(m-n), \\
N(l, m, n)=\left|\frac{2^{l}(2 \Sigma-1) !}{l !(2 \Sigma+l-1) !}\right|^{\frac{1}{2}}, & \Sigma=\bar{\Sigma}+\frac{1}{2}(m+n) .
\end{array}
$$

With these norm factors the matrix elements of the $S p(4, R)$ generators can be given in general algebraic form. The matrix elements of the $\operatorname{Sp}(2, R)$ generators are well known

$$
\left\langle\bar{A} \bar{\Sigma} A M_{A} \pm 1 \Sigma M_{\Sigma}\left|\Lambda_{ \pm}\right| \bar{\Lambda} \bar{\Sigma} A M_{A} \Sigma M_{\Sigma}\right\rangle= \pm \sqrt{\frac{1}{2}} \sqrt{M_{A}\left(M_{A} \pm 1\right)-A(A-1)}
$$

and similarly for $\Sigma_{+}$.

By inverting eqs. (26), the four operators $B_{z x}^{\dagger}, B_{z x}, A_{z x}$ and $A_{x z}$ can be expressed in terms of $O_{++}, A_{+} O_{-+}, \Sigma_{+} O_{+-}, A_{+} \Sigma_{+} O_{--}, \ldots$. From these expressions their matrix elements follow. These matrix elements are written most economically in terms of a generalized Wigner-Eckart theorem $\left.{ }^{25}\right)$ for $\operatorname{Sp}(4, \mathrm{R})$ by using their $T^{\frac{1}{2} \frac{1}{2}}$ tensor property, [see eqs. (20)]

$$
\begin{aligned}
& \left\langle\bar{A} \bar{\Sigma} \Lambda^{\prime} M_{A}^{\prime} \Sigma^{\prime} M_{\Sigma}^{\prime}\left|T_{\tilde{M}_{A}}^{\frac{1}{2}} \frac{1}{\tilde{M}}\right| \bar{\Lambda} \bar{\Sigma} A M_{A} \Sigma M_{\Sigma}\right\rangle=\left\langle\bar{A} \bar{\Sigma} A^{\prime} \Sigma^{\prime}\left\|T^{\frac{1}{2}}\right\| \bar{\Lambda} \bar{\Sigma} A \Sigma\right\rangle \\
& \times\left\langle A M_{A} \frac{1}{2} \tilde{M}_{A} \mid A^{\prime} M_{A}^{\prime}\right\rangle_{\mathrm{Sp}(2, \mathrm{R})}\left\langle\Sigma M_{\Sigma} \frac{1}{2} \tilde{M}_{\Sigma} \mid \Sigma^{\prime} M_{\Sigma}^{\prime}\right\rangle_{\mathrm{Sp}(2, \mathbf{R})},
\end{aligned}
$$

where $\Lambda^{\prime}\left(\Sigma^{\prime}\right)=\Lambda \pm \frac{1}{2}\left(\Sigma \pm \frac{1}{2}\right)$. The $\mathrm{Sp}(2, \mathrm{R})$ Wigner coefficients involve the coupling of a non-unitary (finite-dimensional) irrep with one that is unitary to make another unitary representation. These coupling coefficients are found to be equal (up to phase) to the ones derived by $U^{15}{ }^{15}$ ) for the isomorphic algebra $S U(1,1)$. The results are summarized in tables 2 and 3 . It is interesting to compare these results with those for the compact case. The $\operatorname{Sp}(2, R)$ Wigner coefficients for the coupling $J \otimes \frac{1}{2}$ can be obtained from those for $\mathrm{SU}(2)$ by making the replacement $J \rightarrow-J$; along with appropriate changes in overall phase. A similar correspondence exists for the reduced matrix elements ${ }^{26}$ ) for the compact unitary symplectic group $\mathrm{US}_{\mathrm{p}_{4}} \supset \mathrm{US}_{\mathrm{p}_{2}} \times \mathrm{US}_{\mathrm{p}_{2}}$.

The matrix elements of $A_{z x}, A_{x z}$ are vital in constructing states of good $\mathrm{SU}(3) \supset \mathrm{SU}(2) \times \mathrm{U}(1)$ symmetry in terms of linear combinations of the states 
TABLE 2

Reduced matrix elements of $T^{1 / 21 / 2}$

\begin{tabular}{|c|c|c|}
\hline$\Lambda^{\prime}$ & $\Sigma^{\prime}$ & $\left\langle\bar{\Lambda} \bar{\Sigma} \Lambda^{\prime} \Sigma^{\prime}\left\|T^{\frac{1}{2}} \frac{1}{2}\right\| \bar{\Lambda} \bar{\Sigma} \Lambda \Sigma\right\rangle$ \\
\hline \multirow{2}{*}{$A+\frac{1}{2}$} & \multirow{2}{*}{$\Sigma+\frac{1}{2}$} & {$[(\Lambda+\lambda+\Sigma-\Sigma)(\Lambda-\lambda+\Sigma+\Sigma-1)(\Lambda-\lambda+\Sigma-\Sigma+1)(\Lambda+\lambda+\Sigma+\Sigma-2)]^{ \pm}$} \\
\hline & & $(2 \Lambda)(2 \Sigma)$ \\
\hline \multirow{2}{*}{$A-\frac{1}{2}$} & \multirow{2}{*}{$\Sigma+\frac{1}{2}$} & {$[(\bar{\Lambda}-\Lambda+\Sigma-\bar{\Sigma}+1 \times \bar{\Lambda}+\Lambda-\Sigma+\bar{\Sigma}-2)(\bar{\Lambda}+\Lambda-\Sigma-\bar{\Sigma})(\bar{\Lambda}-\Lambda+\Sigma+\bar{\Sigma}-1)]^{\frac{1}{2}}$} \\
\hline & & $(2 \Lambda-2)(2 \Sigma)$ \\
\hline \multirow{2}{*}{$A+\frac{1}{2}$} & \multirow{2}{*}{$\Sigma-\frac{1}{2}$} & {$[(\bar{\Lambda}-\Lambda+\Sigma-\bar{\Sigma})(\bar{\Lambda}+\Lambda-\Sigma+\bar{\Sigma}-1)(\Lambda+\bar{\Lambda}-\Sigma-\bar{\Sigma}+1)(\bar{\Lambda}-\Lambda+\Sigma+\bar{\Sigma}-2)]^{\frac{1}{2}}$} \\
\hline & & $(2 \Lambda)(2 \Sigma-2)$ \\
\hline \multirow{2}{*}{$A-\frac{1}{2}$} & \multirow{2}{*}{$\Sigma-\frac{1}{2}$} & {$[(\Lambda-\bar{\Lambda}+\Sigma-\bar{\Sigma})(\Lambda+\bar{\Lambda}+\Sigma+\bar{\Sigma}-3)(\Lambda-\bar{\Lambda}+\Sigma+\bar{\Sigma}-2)(\Lambda+\bar{\Lambda}+\Sigma-\bar{\Sigma}-1)]^{\frac{1}{2}}$} \\
\hline & & $(2 \Lambda-2)(2 \Sigma-2)$ \\
\hline
\end{tabular}

TABLE 3

$\mathrm{Sp}(2, \mathrm{R})$ Wigner coefficients for $J \otimes \frac{1}{2}$

\begin{tabular}{ccc}
$J^{\prime}$ & $\tilde{M}_{J}$ & $\left\langle J M_{J} \frac{1}{2} \tilde{M}_{J} \mid J^{\prime} M_{J}^{\prime}\right\rangle$ \\
\hline$J+\frac{1}{2}$ & $\frac{1}{2}$ & $\sqrt{\frac{J+M_{J}}{2 J-1}}$ \\
$J+\frac{1}{2}$ & $-\frac{1}{2}$ & $\sqrt{\frac{M_{J}-J}{2 J-1}}$ \\
$J-\frac{1}{2}$ & $\frac{1}{2}$ & $\sqrt{\frac{\bar{M}_{J}-\bar{J}+1}{2 J-1}}$ \\
$J-\frac{1}{2}$ & $-\frac{1}{2}$ & $\sqrt{\frac{J+M_{J}-1}{2 J-1}}$
\end{tabular}

$\left|\bar{\Lambda} \bar{\Sigma} A M_{A} \Sigma M_{\Sigma}\right\rangle \equiv|\bar{\Lambda} \bar{\Sigma} k l m n\rangle \equiv|k l m n\rangle$ of eq. (28). The SU(3) ladder operators $A_{z y}$, $A_{x y}$ automatically annihilate the states $|k l m n\rangle$. An SU(3) highest weight state is therefore obtained from the condition

$$
A_{z x}|(\lambda \mu) \mathrm{HW}\rangle=\sum_{k l m n} A_{z x}|k l m n\rangle\langle k l m n \mid(\lambda \mu) \mathrm{HW}\rangle=0 .
$$

The number of terms in the sum of eq. (35), and consequently the number of coefficients $\langle k|m n| \mathrm{HW}\rangle$, is equal to the degeneracy of the weight $\left(M_{A}, M_{\Sigma}\right)$ with

$$
\begin{gathered}
M_{A}-\bar{\Lambda}=\frac{1}{2}\left[N_{z}+\frac{1}{2}(A-1)\right]-\frac{1}{2}\left[\lambda_{0}+\mu_{0}+v_{0}+\frac{1}{2}(A-1)\right]=\frac{1}{2}\left(\lambda+\mu-\lambda_{0}-\mu_{0}\right), \\
M_{\Sigma}-\bar{\Sigma}=\frac{1}{2}\left[N_{x}+\frac{1}{2}(A-1)\right]-\frac{1}{2}\left[\mu_{0}+v_{0}+\frac{1}{2}(A-1)\right]=\frac{1}{2}\left(\mu-\mu_{0}\right),
\end{gathered}
$$

where $N_{z}=\lambda+\mu+v, N_{x}=\mu+v$, the number of squares in the 1 st and 2 nd rows of 
the tableau for $(\lambda \mu)$, respectively, and $v=v_{0}$. The degeneracy of the weight $\left(M_{A}, M_{\Sigma}\right)$, to be denoted by $\rho_{\mathrm{m}}\left(M_{A}, M_{\Sigma}\right)$, is equal to the number of solutions $(k, l, m, n)$ to the two equations

$$
\begin{aligned}
& M_{A}=\bar{\Lambda}+k+\frac{1}{2}(m-n), \\
& M_{\Sigma}=\bar{\Sigma}+l+\frac{1}{2}(m+n) .
\end{aligned}
$$

Since the $\mathrm{SU}(3)$ ladder operator, $A_{z x}$, increases $M_{A}$ by $+\frac{1}{2}$ and decreases $M_{\Sigma}$ by $-\frac{1}{2}$, the vector $\left.\left.\left(A_{z x}\right)(\lambda \mu) \mathrm{HW}\right\rangle\right)$ of eq. (35) can be expressed as a linear combination of $\rho_{\mathrm{m}}\left(M_{A}+\frac{1}{2}, M_{\Sigma}-\frac{1}{2}\right)=\rho_{\mathrm{m}}\left(\bar{\lambda}+\frac{1}{2}\left(\lambda+\mu-\lambda_{0}-\mu_{0}+1\right), \bar{\Sigma}+\frac{1}{2}\left(\mu-\mu_{0}-1\right)\right)$ independent vectors and thus leads to a number of conditions which is equal to this $\rho_{\mathrm{m}}$. The number of independent states $(\lambda \mu)$, i.e., the number of independent solutions to eq. (35), is to be denoted by $\gamma_{m}(\lambda \mu)$, (the maximum value of the label $\gamma$ of eq. $(11) ; \gamma=1,2, \ldots$, $\left.\gamma_{\mathrm{m}}\right) \ldots$ Thus

$$
\begin{aligned}
\gamma_{\mathrm{m}}(\hat{\lambda} \mu)=\rho_{\mathrm{m}}\left(\bar{\Lambda}+\frac{1}{2}\left(\hat{\lambda}+\mu-\hat{\lambda}_{0}-\mu_{0}\right), \bar{\Sigma}+\frac{1}{2}\left(\mu-\mu_{0}\right)\right) \\
\\
-\rho_{\mathrm{m}}\left(\bar{\Lambda}+\frac{1}{2}\left(\hat{\lambda}+\mu-\hat{\lambda}_{0}-v_{0}+1\right), \bar{\Sigma}+\frac{1}{2}\left(\mu-\mu_{0}-1\right)\right) .
\end{aligned}
$$

The weight space diagram with the degeneracies of $\left(M_{A}, M_{\Sigma}\right)$ fully displayed are thus of central importance. Fig. 2 shows such a weight space diagram for the symplectic irrep most relevant for the ground-state rotational band in ${ }^{24} \mathrm{Mg}$ as well as the rotational band based on the $6.05 \mathrm{MeV} 0^{+}$state in ${ }^{16} \mathrm{O}$. Both of these are dominated by $\mathrm{SU}(3)$ irreps with $(\lambda \mu)=(84)$ and lead to symplectic irreps with $\left\{\lambda_{0} \mu_{0} v_{0}\right\}=\{840\}$ for ${ }^{16} \mathrm{O}$ and $\{844\}$ for ${ }^{24} \mathrm{Mg}$. Fig. 2 is a magnification of fig. 1 for this special case, where the origin has been shifted to the point $\left(M_{A}, M_{\Sigma}\right)=(\bar{A}, \bar{\Sigma})$. States on the solid lines, with $M_{A}+M_{\Sigma}=$ constant, correspond to shell-model core excitations with an excitation energy of $2 N \hbar \omega$, with $N=k+l+m$; see eq. (28). The weights $\left(M_{A}, M_{\Sigma}\right)$ are labeled with the symbols $(\lambda \mu)_{\rho_{\mathrm{m}}}^{\lambda_{\mathrm{m}}}$, where $(\lambda \mu)$ are related to $M_{\Lambda}-\bar{\Lambda}, M_{\Sigma}-\bar{\Sigma}$ by eq. (36). E.g., the weight point $M_{A}-\bar{\Lambda}=2, M_{\Sigma}-\bar{\Sigma}=1$, is labeled by $(10,6)_{4}^{2}$ in fig. 2 . This point can be reached from the state $M_{A}-\bar{A}=0, M_{\Sigma}-\bar{\Sigma}=0$, by 4 independent operators with $(k, l, m, n)=(3,0,0,2),(2,0,1,1),(2,1,0,0)$ and $(1,0,2,0)$, so that the degeneracy for this weight is $\rho_{\mathrm{m}}=4$. The HW state for $N=3,(\lambda \mu)=(10,6)$ must be constructed from a linear combination of these 4 states. Note also that the degeneracy of the weight point $M_{A}-\bar{A}=\frac{5}{2}, M_{\Sigma}-\bar{\Sigma}=\frac{1}{2}$ is 2 , corresponding to $(k, l, m, n)=(3,0,0,1),(2,0,1,0)$ so that $\gamma_{m}=2(=4-2)$. There are therefore two independent states with $(\lambda \mu)=(10,6)$ in the irreps $\left\{84 v_{0}\right\}$.

The simplest states are those with $M_{\Sigma}=\bar{\Sigma}(l=m=n=0)$, which are reached by $k=N$ actions of the operator $\Lambda_{+}$. Since $\Lambda_{+}$is proportional to the operator $O_{z z}^{\dagger}$ of eq. (16), the states with $M_{\Sigma}=\bar{\Sigma}$ satisfy eq. (35) automatically and are SU(3) highest weight states

$$
\left|\bar{\Lambda} \bar{\Sigma} \bar{\Lambda}\left(M_{A}=\bar{\Lambda}+N\right) \bar{\Sigma} \bar{\Sigma}\right\rangle=\left|\left\{\lambda_{0} \mu_{0} v_{0}\right\}\left(\lambda_{0}+2 N, \mu_{0}\right) v_{0} \mathrm{HW}\right\rangle
$$




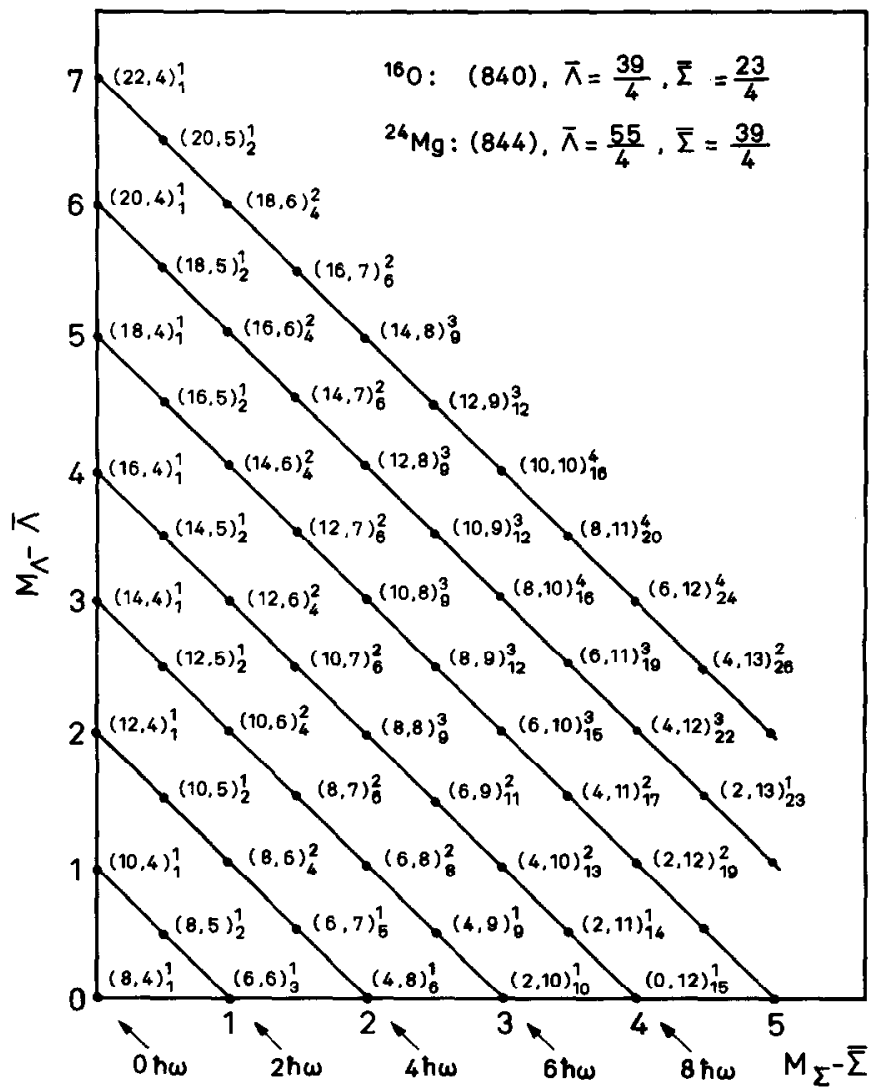

Fig. 2. Weight space diagram for (84) bands in ${ }^{16} \mathrm{O}$ and ${ }^{24} \mathrm{Mg}$.

Note that $\rho_{\mathrm{m}}\left(M_{A}=\bar{\lambda}+N, M_{\Sigma}=\bar{\Sigma}\right)=\gamma_{\mathrm{m}}\left(\lambda_{0}+2 N, \mu_{0}\right)=1$; and that these states along the $M_{A}$ axis, labeled $\left(\lambda_{0}+2 N, \mu_{0}\right)_{1}^{1}$, constitute the $\operatorname{Sp}(2, \mathbf{R})_{2}$ model of Arickx $\left.{ }^{11}\right)$.

Now consider the weights $\left(M_{A}, M_{\Sigma}\right)=\left(\bar{\Lambda}+N-\frac{1}{2}, \bar{\Sigma}+\frac{1}{2}\right)$, with degeneracy $\rho_{\mathrm{m}}=2$. The two states possessing this weight are $|k, l, m, n\rangle=|N-1,0,1,0\rangle$ and $|N, 0,0,1\rangle$. One linear combination of these two states is the state

$$
\begin{aligned}
\mid\left(\lambda_{0}+2 N, \mu_{0}\right) \varepsilon=2\left(\lambda_{0}+2 N\right)+\mu_{0}-3, M_{\tilde{\Lambda}} & \left.=\tilde{\Lambda}=\mu_{0}+\frac{1}{2}\right\rangle \\
= & {\left[\lambda_{0}+2 N\right]^{-\frac{1}{2}} A_{x z}\left|\left(\lambda_{0}+2 N, \mu_{0}\right) \mathrm{HW}\right\rangle }
\end{aligned}
$$

reached by the SU(3) lowering operator $A_{x z}$, with norm factor $\left.{ }^{7,24}\right)\left[\lambda_{0}+2 N\right]^{-\frac{1}{2}}$. The matrix elements of $A_{x z}$ can be read from tables 2 and 3. The linear combination of $|N-1,0,1,0\rangle=\left|\bar{\Lambda} \bar{\Sigma}, \bar{\Lambda}+\frac{1}{2}, \bar{\Lambda}+N-\frac{1}{2}, \bar{\Sigma}+\frac{1}{2}, \bar{\Sigma}+\frac{1}{2}\right\rangle$ and $|N, 0,0,1\rangle=\mid \bar{\Lambda} \bar{\Sigma}, \bar{\Lambda}-\frac{1}{2}$, $\left.\bar{\Lambda}+N-\frac{1}{2}, \bar{\Sigma}+\frac{1}{2}, \bar{\Sigma}+\frac{1}{2}\right\rangle$, orthogonal to the state of eq. (40), is the solution to eq. (35) which gives the HW state for $(\lambda \mu)=\left(\lambda_{0}+2 N-2, \mu_{0}+1\right)$. From tables 2 and 3 this 
state is given by

$$
\begin{aligned}
\mid\left(\lambda_{0}+2 N-2, \mu_{0}+1\right) & \left.v_{0} \mathrm{HW}\right\rangle= \\
& {\left[\frac{(2 \bar{\Lambda}+N-1)(\bar{\Lambda}-\bar{\Sigma})}{(\bar{\Lambda}-\bar{\Sigma}+N)(2 \bar{\Lambda}-1)}\right]^{\frac{1}{2}}\left|\bar{\Lambda} \bar{\Sigma} \bar{\Lambda}+\frac{1}{2} \bar{\Lambda}+N-\frac{1}{2} \bar{\Sigma}+\frac{1}{2} \bar{\Sigma}+\frac{1}{2}\right\rangle } \\
& -\left[\frac{N(\bar{\Lambda}+\bar{\Sigma}-1)}{(\bar{\Lambda}-\bar{\Sigma}+N)(2 \bar{\Lambda}-1)}\right]^{\frac{1}{2}}\left|\bar{\Lambda} \bar{\Sigma} \bar{\Lambda}-\frac{1}{2} \bar{\Lambda}+N-\frac{1}{2} \bar{\Sigma}+\frac{1}{2} \bar{\Sigma}+\frac{1}{2}\right\rangle
\end{aligned}
$$

The $\mathrm{SU}(3)$ reduced matrix elements of the $\mathrm{Sp}(6, \mathrm{R})$ raising operators, $T^{(20)}$, between the two classes of states given by eqs. (39) and (41), follow from eq. (33), tables 2 and 3 , and some simple $\mathrm{SU}(3) \supset \mathrm{SU}(2) \times \mathrm{U}(1)$ Wigner coefficients ${ }^{24}$ ) to yield

$$
\begin{array}{r}
\left\langle\left(\lambda_{0}+2 N+2, \mu_{0}\right) v_{0}\left\|T^{(20)}\right\|\left(\lambda_{0}+2 N, \mu_{0}\right) v_{0}\right\rangle=[2(N+1)(N+2 \bar{\Lambda})]^{\frac{1}{2}}, \\
\left\langle\left(\lambda_{0}+2 N, \mu_{0}+1\right) v_{0}\left\|T^{(20)}\right\|\left(\hat{\lambda}_{0}+2 N, \mu_{0}\right) v_{0}\right\rangle=\left[\begin{array}{c}
2(\bar{\Lambda}-\bar{\Sigma})(\bar{\Lambda}+\bar{\Sigma}-1) \\
(\overline{\bar{A}}-\bar{\Sigma}+N)
\end{array}\right]^{\frac{1}{2}}, \\
\left\langle\left(\lambda_{0}+2 N, \mu_{0}+1\right) v_{0}\left\|T^{(20)}\right\|\left(\lambda_{0}+2 N-2, \mu_{0}+1\right) v_{0}\right\rangle \\
=\left[\frac{2 N(2 \bar{A}+N-1)(\bar{\Lambda}-\bar{\Sigma}+N+1)}{(\bar{A}-\bar{\Sigma}+N)}\right]^{\frac{1}{2}}
\end{array}
$$

A straightforward generalization of this procedure can be used to construct the states with $\left|\left(\lambda_{0}+2 N-2 n, \mu_{0}+n\right) \mathrm{HW}\right\rangle, n=2,3, \ldots$, and the SU(3) lower weight states reached by acting on such highest weight states with $p \mathrm{SU}(3)$ lowering operators, $A_{x z}$. These (normalized) states will be denoted by $\left\{\left\{\lambda_{0} \mu_{0} v_{0}\right\}(\lambda \mu) v_{0} p \gamma^{\prime}\right\rangle$. With the construction of these states, the operator, $T_{400}^{(20)}=2 A_{+}$, is sufficient to calculate SU(3) reduced matrix elements via the Wigner-Eckart theorem and eq. (33):

$$
\begin{aligned}
& \left\langle\left\{\lambda_{0} \mu_{0} v_{0}\right\}\left(\lambda^{\prime} \mu^{\prime}\right) v_{0} p^{\prime} \gamma^{\prime}\left\|T^{(20)}\right\|\left\{\lambda_{0} \mu_{0} v_{0}\right\}(\lambda \mu) v_{0} p^{\prime}\right\rangle \times\left\langle(20) 40 ;(\lambda \mu) \varepsilon(p) \tilde{\Lambda}(p) \|\left(\lambda^{\prime} \mu^{\prime}\right) \varepsilon^{\prime}\left(p^{\prime}\right) \tilde{\Lambda}\left(p^{\prime}\right)\right\rangle \\
& \quad=\sum_{k \operatorname{lm} n}\left\langle\left\{\lambda_{0} \mu_{0} v_{0}\right\}\left(\lambda^{\prime} \mu^{\prime}\right) v_{0} p^{\prime} \gamma^{\prime} \mid k+1 \operatorname{lm} n\right\rangle\left\langle k \operatorname{lm} n \mid\left\{\lambda_{0} \mu_{0} v_{0}\right\}(\lambda \mu) v_{0} p \gamma^{\prime}\right\rangle \\
& \quad \times[2(k+1)(2 \bar{\Lambda}+m-n+k)]^{\frac{1}{2}} .
\end{aligned}
$$

Here the $\langle k|m n| \ldots\rangle$ are the expansion coefficients of the states with good SU(3) quantum numbers, see eq. (35); and $\langle\|\rangle$ is an $\mathrm{SU}(3) \supset \mathrm{SU}(2) \times \mathrm{U}(1)$ Wigner coefficient ${ }^{18,24}$ ), with $\delta(p)=2 \lambda+\mu-3 p, \widetilde{\Lambda}(p)=\frac{1}{2}(\mu+p)$.

\section{The $S p(4, R)$ model of collective motion}

As a prelude to more realistic structure studies it is instructive to examine the idealization in which $\operatorname{Sp}(6, \mathrm{R})$ is treated as an exact symmetry by restricting the model space to a single unitary irreducible representation of this algebra. This may be a good approximation in nuclei such as ${ }^{24} \mathrm{Mg}$ where conventional shell-model studies 
[refs. $\left.\left.{ }^{27,28}\right)\right]$ indicate that the ground-state rotational band is dominated by a single $\mathrm{SU}(3)$ representation, with $(\lambda \mu)=(84)$. The symplectic band $\left\{\lambda_{0} \mu_{0} v_{0}\right\}=\{844\}$ built on this particular shell-model state should thus give a good description of the core excitations required to account for the observed quadrupole collectivity in this nucleus. Since symplectic symmetry will not be broken, the hamiltonian, (constructed from the bare $G$-matrix elements, e.g.), can be projected onto a symmetry-preserving hamiltonian. Such a hamiltonian can be constructed from the generators of the svmplectic algebra. We therefore follow Rosensteel and Rowe ${ }^{2,3,17}$ ) and use the symmetry-preserving hamiltonian

$$
\begin{gathered}
H_{\text {collective }}=H_{0}+U\left(\left[Q^{(2)}\right]\right), \\
H_{0} \equiv \sum_{\alpha=1}^{A}\left\{\frac{1}{2 m} p^{\prime 2}(\alpha)+\frac{1}{2} m \omega_{0}^{2} r^{\prime 2}(\alpha)\right\},
\end{gathered}
$$

with

$$
\begin{gathered}
U\left(\left[Q^{(2)}\right]\right)=A_{2} \operatorname{Tr}\left(Q^{2}\right)+A_{3} \operatorname{Tr}\left(Q^{3}\right)+A_{4}\left(\operatorname{Tr}\left(Q^{2}\right)\right)^{2}, \\
\operatorname{Tr}\left(Q^{2}\right)=\frac{8}{3} \sqrt{\frac{1}{5}} \pi\left[Q^{(2)} \times Q^{(2)}\right]^{(0)}, \\
\operatorname{Tr}\left(Q^{3}\right)=-\frac{16}{45} \pi \sqrt{\frac{7}{2}} \pi\left[Q^{(2)} \times Q^{(2)} \times Q^{(2)}\right]^{(0)} .
\end{gathered}
$$

The square brackets denote angular momentum coupling. $Q^{(2)}$ is given in spherical tensor form by

$$
\begin{gathered}
Q_{m}^{(2)} \equiv \sum_{\alpha=1}^{A} r^{\prime 2}(\alpha) Y_{m}^{(2)}(\hat{\Omega} \alpha) . \\
r^{\prime}(\alpha) \equiv r(\alpha)-R_{\text {c.m. }}, \quad p^{\prime}(\alpha) \equiv p(\alpha)-\frac{1}{A} P_{\text {c.m. }} .
\end{gathered}
$$

The three terms in the potential, $U$, are the shell model analog of a collective potential given in terms of Bohr-Mottelson ${ }^{1}$ ) deformation parameters $\beta$ and $\gamma$

$$
V(\beta, \gamma)=\tilde{C}_{2} \beta^{2}+\tilde{C}_{3} \beta^{3} \cos 3 \gamma+\tilde{C}_{4} \beta^{4} .
$$

To evaluate the matrix elements of this hamiltonian it is important to expand $U\left(Q^{(2)}\right)$ in terms of coupled $\mathrm{SU}(3)$ irreducible tensor operators. The operator $Q^{(2)}$ can be written in terms of $\operatorname{Sp}(6, \mathrm{R})$ generators as

$$
Q_{M}^{(2)}=(i)^{2+M} \sqrt{15 / 4 \pi}\left(h / 2 m \omega_{0}\right)\left\{T_{12 M}^{(20)}+2 T_{12 M}^{(11)}+T_{12 M}^{(02)}\right\},
$$

where the spherical tensor operators are related to those in the $S U(2) \times U(1)$ basis by the transformation coefficients of Akiyama and Draayer ${ }^{18}$ );

$$
T_{\kappa L M}^{(\lambda \mu)}=\sum_{\varepsilon \tilde{\tilde{A} M} \tilde{\Lambda}}\left\langle(\lambda \mu) \varepsilon \tilde{\Lambda} M_{\tilde{\Lambda}} \tilde{\|}(\lambda \mu) \kappa L M\right\rangle T_{\varepsilon \tilde{A} M \tilde{\pi}}^{(\lambda \mu)}
$$

Before proceeding it is convenlent to introduce the following notation. Let $\Gamma$ denote 
the $\mathrm{Sp}(6, \mathrm{R})$ and $\mathrm{SU}(3)$ irrep labels; while $\eta^{\mathrm{s}}$ and $\eta^{\mathrm{c}}$ are to refer to the $\mathrm{O}_{3}^{+} \supset \mathrm{O}_{2}^{+}$ and $S U(2) \times U(1)$ subgroup labels respectively

$$
\Gamma \equiv\left\{\lambda_{0} \mu_{0} v_{0}\right\}(\lambda \mu), \quad \eta^{\mathrm{s}} \equiv \kappa L M, \quad \eta^{\mathrm{c}} \equiv \varepsilon \tilde{\Lambda} M_{\tilde{\Lambda}}
$$

so that a general symplectic state is denoted by $|\Gamma v \eta \gamma\rangle$, cf. eq. (11). In this notation, the Wigner-Eckart theorem can be used to define the $\mathrm{SU}(3)$ reduced matrix elements

$$
\left\langle\Gamma^{\prime} v^{\prime} \eta^{\prime} \gamma^{\prime}\left|T_{\bar{\eta}}^{\bar{\Gamma}}\right| \Gamma v \eta \gamma\right\rangle=\sum_{\rho}\left\langle\bar{\Gamma} \bar{\eta} \Gamma \eta \mid \Gamma^{\prime} \eta^{\prime}\right\rangle_{\rho}\left\langle\Gamma^{\prime} v^{\prime} \gamma^{\prime} \| T^{\bar{\Gamma}}|| \Gamma v \gamma\right\rangle_{\rho} .
$$

Note the unconventional ordering in the Wigner coefficient. This order has been chosen to simplify expressions for the reduced matrix elements of coupled tensor operators. The more common choice would have been $\left\langle\Gamma \eta \bar{\Gamma} \bar{\eta} \mid \Gamma^{\prime} \eta^{\prime}\right\rangle_{\rho}$. The sum over multiplicity labels $\rho$ is not needed for the matrix elements of $\operatorname{Sp}(6, R)$ generators. With $\bar{\Gamma}=(20)$ or $(02)$, the products are multiplicity-free. The $T^{(11)}$ tensors are SU(3) generators. These have the property $\left.{ }^{24}\right)\left\langle\left\|T^{(11)}\right\|\right\rangle_{\rho=2}=0$. The reduced matrix element of a $T^{(11)}$ generator is given by

$$
\begin{aligned}
& \left\langle\Gamma^{\prime} v^{\prime} \gamma^{\prime}\left\|T^{(11)}\right\| \Gamma v_{0} \gamma^{\prime}\right\rangle \\
& \quad=\delta_{\Gamma^{\prime} T^{\prime} v^{\prime} \gamma_{\gamma^{\prime} \gamma}} \delta\left[-\frac{1}{2} \sqrt{\frac{1}{3}}(2 i+\mu)\right]\left\langle(11) 00(\lambda \mu) \varepsilon_{\mathrm{HW}} \tilde{\Lambda}_{\mathrm{HW}} \|(\lambda \mu) \varepsilon_{\mathrm{HW}} \tilde{\Lambda}_{\mathrm{HW}}\right\rangle_{\rho=1}^{-1} .
\end{aligned}
$$

The reduced matrix eleements of $T^{(20)}$ operators in the $\operatorname{Sp}(4, \mathrm{R})$ model have been given by eq. (43). Finally, using the conjugation property, eq. (8), and symmetry relations for the $\mathrm{SU}(3)$ Wigner coefficients ${ }^{18}$ )

$$
\begin{aligned}
& \left\langle\Gamma^{\prime} v_{0} \gamma^{\prime}\left\|T^{(02)}\right\| \Gamma v_{0} \gamma\right\rangle \\
& \quad=(-1)^{\theta(\Gamma)-\theta\left(\Gamma^{\prime}\right)} \sqrt{\operatorname{dim}(\Gamma) / \operatorname{dim}\left(\Gamma^{\prime}\right)}\left\langle\Gamma v_{0} \gamma\left\|T^{(20)}\right\| \Gamma^{\prime} v_{0} \gamma^{\prime}\right\rangle,
\end{aligned}
$$

where $\theta(\Gamma) \equiv \lambda+\mu$. With these $\mathrm{SU}(3)$ reduced matrix elements, the matrix elements of $\mathrm{U}\left(Q^{(2)}\right)$ can be calculated by $\mathrm{SU}(3)$ recoupling techniques. However, it is important to "normal-order" the $\mathrm{SU}(3)$ tensors in the operators $\operatorname{Tr}\left(Q^{\alpha}\right), \alpha=2,3,4$.

$$
\begin{aligned}
& \left.\operatorname{Tr}\left(Q^{x}\right)=\sum_{n^{1} n^{2} n^{3} n^{4}} f^{\alpha^{1} n^{2} n^{3} n^{4}} \prod_{i j k l} T_{\eta_{i}}^{(20)}\right)^{n_{i}^{i}}\left(T_{\eta_{j}}^{(02)}\right)^{n_{j}^{2}}\left(T_{\eta_{k}}^{(11)}\right)^{n_{k}^{3}}\left(T^{(00)}\right)^{n^{4}} . \\
& n_{1}^{1}+\ldots+n_{8}^{3} \leq x
\end{aligned}
$$

In applications restricted to the $S p(4, R)$ model it is important that the action of the operators $T^{(02)}$ precede that of the raising operators $T^{(20)}$. An operator $T^{(02)}$ acting on an excited state of a symplectic band will in general create states $\left|\left\{\lambda_{0} \mu_{0} v_{0}\right\}(\lambda \mu) v \eta \gamma\right\rangle$ with $v \neq v_{0}$, i.e., states outside the $\operatorname{Sp}(4, \mathbf{R})$ model space. However, $T^{(02)}$ annihilates the states $\left|\left\{\hat{\lambda}_{0} \mu_{0} v_{0}\right\}\left(\lambda_{0} \mu_{0}\right) v_{0} \eta 1\right\rangle$, so that the action of the operators (58) preserve the $\operatorname{Sp}(4, R)$ symmetry.

To evaluate matrix elements of $\operatorname{Tr}\left(Q^{\alpha}\right)$ it is convenient to express these in terms 
of SU(3) coupled tensors

$$
\operatorname{Tr}\left(Q^{2}\right)=2 \sqrt{5} \sum_{\Gamma_{1} \Gamma_{2} \Gamma} C\left(\Gamma_{1}\right) C\left(\Gamma_{2}\right)\left\langle\Gamma_{1} 12 ; \Gamma_{2} 12 \| \Gamma 10\right\rangle\left[T^{\Gamma_{1}} \times T^{\Gamma_{2}}\right]_{100}^{\Gamma_{10}},
$$

where the square bracket denotes $\mathrm{SU}(3)$ coupling, where $C(\Gamma) \equiv\left(1+\delta_{\Gamma(11)}\right)$ accounts for the factor 2 in eq. (52), and where $\left\langle\Gamma_{1} \kappa_{1} L_{1} ; \Gamma_{2} \kappa_{2} L_{2} \| \Gamma \kappa L\right\rangle_{p}$ is an $\mathrm{SU}(3) \supset 0_{3}^{+}$ Wigner coefficient ${ }^{18}$ ). For the operator, quadratic in $Q$, only the label $\rho=1$ is needed. The coupled operators in eq. (59) can be put into "normal-ordered form" with the use of commutators $\left[T_{\eta_{1}}^{\Gamma_{1}}, T_{\eta_{2}}^{\Gamma_{2}}\right]$, where needed. Finally defining

$$
\operatorname{Tr}\left(Q^{\alpha}\right)=\widetilde{\operatorname{Tr}}\left(Q^{\alpha}\right)+\left(\widetilde{\operatorname{Tr}}\left(Q^{\alpha}\right)\right)^{\dagger}
$$

the quadratic term can be put in the desired form

$$
\begin{aligned}
& \operatorname{Tr}\left(Q^{2}\right)=\frac{4}{3} \sqrt{5} S_{(20)(20)}^{(40)}+\frac{10}{3} S_{(20)(20)}^{(02)}+8 \sqrt{5} S_{(20)(11)}^{(20)}+\frac{1}{3} \sqrt{30} S_{(20)(02)}^{(22)} \\
&-\frac{40}{3} C_{\mathrm{Sp}(6 . \mathrm{R})}^{(2)}+17 C_{\mathrm{SU}(3)}^{(2)}+\frac{5}{18} N^{2}-L^{2}-\frac{20}{3} \sqrt{6} T_{L=0}^{(20)},
\end{aligned}
$$

where

$$
S_{\Gamma_{1} \Gamma_{2}}^{\Gamma_{*} \rho} \equiv\left[T^{r_{1}} \times T^{\Gamma_{2}}\right]_{100}^{\Gamma, \rho}
$$

Similarly,

$$
\begin{aligned}
\widetilde{\operatorname{Tr}}\left(Q^{3}\right)= & \sum_{\substack{\Gamma_{1} \Gamma_{2} \Gamma_{3} \\
\Gamma_{12} \rho_{12} \Gamma \rho}}^{t} \Delta_{3}\left(\Gamma_{1} \Gamma_{2} \Gamma_{3} ; \Gamma_{12} \rho_{12} ; \Gamma \rho\right) R_{\Gamma_{1} \Gamma_{2}: \Gamma_{3}}^{\Gamma_{12} \rho_{12} \Gamma_{\rho}} \\
& +\sum_{\Gamma_{1} \Gamma_{2} \Gamma_{\rho}}^{\prime} \Delta_{2}\left(\Gamma_{1} \Gamma_{2} ; \Gamma \rho\right) S_{\Gamma_{1} \Gamma_{2}}^{\Gamma_{\rho}}+\sum_{\Gamma} \Delta_{1}(\Gamma) T_{100}^{r}
\end{aligned}
$$

where the primes on the summation signs indicate only normal-ordered combinations of $\Gamma_{1} \Gamma_{2} \ldots$ are included. Also

$$
\begin{gathered}
R_{\Gamma_{1} \Gamma_{2} \Gamma_{3} \Gamma_{3}}^{\Gamma_{12} \Gamma_{\rho}} \equiv\left[\left[T^{\Gamma_{1}} \times T^{\Gamma_{2}}\right]_{\rho_{12}}^{\Gamma_{12}} \times T^{\Gamma_{3}}\right]_{100}^{\Gamma_{\rho}} \\
\Delta_{3}\left(\Gamma_{1} \Gamma_{2} \Gamma_{3} ; \Gamma_{12} \rho_{12} ; \Gamma \rho\right)=\sqrt{\frac{70}{3} C\left(\Gamma_{1}\right) C\left(\Gamma_{2}\right) C\left(\Gamma_{3}\right) 0_{3}\left(\Gamma_{1} \Gamma_{2} \Gamma_{3}\right)} \\
\times \sum_{\bar{\kappa}}\left\langle\Gamma_{1} 12 \Gamma_{2} 12 \| \Gamma_{12} \bar{\kappa} 2\right\rangle_{\rho_{12}}\left\langle\Gamma_{12} \bar{\kappa} 2 \Gamma_{3}|2| \mid \Gamma 10\right\rangle_{\rho^{*}}
\end{gathered}
$$

$\theta_{3}\left(\Gamma_{1} \Gamma_{2} \Gamma_{3}\right)$ is equal to the number of ways of permuting $\Gamma_{1}, \Gamma_{2}, \Gamma_{3}$. E.g., $\theta_{3}((20)(20)(11))$ $=3$. If the product is hermitian, however, $\left\{\Gamma_{1} \Gamma_{2} \Gamma_{3}\right\}^{+}=\left\{\Gamma_{1} \Gamma_{2} \Gamma_{3}\right\}$; we must multiply by a factor of $\frac{1}{2}$. E.g., $\theta_{3}((11)(11)(11))=\frac{1}{2}$. The coefficients $\Delta_{2}\left(\Gamma_{1} \Gamma_{2}, \Gamma \rho\right)$ and $\Delta_{1}(\Gamma)$ are obtained in the normal ordering process, and have a form similar to $\Delta_{3}$. The cubic term is summarized in table 4 . The Casimir operators in eq. (61) and table 4 have eigenvalues given by eq. (17) and by

$$
\begin{aligned}
& C_{\mathrm{SU}(3) \text { e.v. }}^{(3)}=(\lambda-\mu)\left[\frac{1}{9}(2 \lambda+\mu)(\lambda+2 \mu)+\lambda+\mu+1\right], \\
& C_{\mathrm{Sp}(6, \mathrm{R})}^{(2)}=\frac{1}{16}\left\{h_{1}\left(h_{1}-2\right)+h_{2}\left(h_{2}-4\right)+h_{3}\left(h_{3}-6\right)\right\},
\end{aligned}
$$


TABLE 4

SU(3) tensor expansion of $\operatorname{Tr}\left(Q^{3}\right)$

\begin{tabular}{|c|c|c|c|}
\hline Tensor & Coefficient & Tensor & Coefficient \\
\hline$R_{(20)(20)(20)}^{(40)(60)}$ & $-\frac{4}{9} \sqrt{14}$ & $R_{(20),(22),(11)}^{(22)}$ & $-8 \sqrt{7}$ \\
\hline$R_{(20)\{20\}(20)}^{(02)(22)}$ & $\frac{7}{5} \sqrt{5}$ & $R_{(20) 1021111)}^{(111122)}$ & $7 \sqrt{3}$ \\
\hline$R_{(20)(20)(20)}^{(02)(00)}$ & $\frac{35}{9}$ & $R_{(20)(02)(11)}^{(1)(00)}$ & $-7 \sqrt{5}$ \\
\hline$R_{(20)(20)(11)}^{(40)(40)}$ & $-\frac{4}{3} \sqrt{105}$ & $R_{(11)(11)(11)}^{(22)(22) \rho=2}$ & $4 \sqrt{7}$ \\
\hline$R_{(20)\{201(11)}^{(02)(02)}$ & $\frac{14}{3} \sqrt{30}$ & $R_{(11), 111(11)}^{(1) p=2(22)}$ & $7 \sqrt{2}$ \\
\hline$R_{(20)(20)(02)}^{(40)(42)}$ & $-\frac{2}{9} \sqrt{210}$ & $C_{\mathrm{SU}(3)}^{(3)}$ & $\frac{7}{4}$ \\
\hline$R_{1201120)(02)}^{(40)(20)}$ & $-\frac{14}{9} \sqrt{15}$ & $S_{12011201}^{(40)}$ & $\frac{28}{3} \sqrt{5}$ \\
\hline$R_{(20)(20)(02)}^{(02)(04)}$ & $\frac{14}{9} \sqrt{30}$ & $S_{(201\{20\}}^{(02)}$ & $\frac{70}{3}$ \\
\hline$R_{(20)(20)(02)}^{102)(20)}$ & $\frac{35}{9} \sqrt{6}$ & $S_{(20)(11)}^{(20)}$ & $35 \sqrt{5}$ \\
\hline$R_{(20)(1) 11(11)}^{(3) 1(42)}$ & $\frac{2}{3} \sqrt{210}$ & $C_{\mathrm{su}(3)}^{(2)}$ & 21 \\
\hline$R_{(20)(11)(11)}^{(12)(04)}$ & $-\frac{14}{3} \sqrt{30}$ & $L^{2}$ & $-\frac{7}{4}$ \\
\hline$R_{(20)(11)(11)}^{(12)(20)}$ & $-\frac{28}{3} \sqrt{15}$ & $T^{(20)}$ & $-\frac{70}{3} \sqrt{6}$ \\
\hline$R_{(20)(111)(11)}^{(20)(20)}$ & $-14 \sqrt{6}$ & $N$ & $\frac{70}{9}$ \\
\hline
\end{tabular}

where $h_{1} \equiv \lambda_{0}+\mu_{0}+v_{0}+\frac{1}{2}(A-1), h_{2}=h_{1}-\lambda_{0}, h_{3}=h_{2}-\mu_{0}$, and also:

$$
N_{\text {e.v. }}=\lambda+2 \mu+3 v+\frac{3}{2}(A-1) \text {. }
$$

The matrix elements of the quartic term can be evaluated from the matrix elements of the quadratic terms via

$$
\left\langle\alpha\left|\left(\operatorname{Tr}\left(Q^{2}\right)\right)^{2}\right| \beta\right\rangle=\sum_{;}\left\langle\alpha\left|\operatorname{Tr}\left(Q^{2}\right)\right| \gamma\right\rangle\left\langle\gamma\left|\operatorname{Tr}\left(Q^{2}\right)\right| \beta\right\rangle .
$$

In the full $\operatorname{Sp}(6, R)$ model this expression is exact; the states $|\gamma\rangle$ range over all subgroup labels of $\left\{\lambda_{0} \mu_{0} v_{0}\right\}$. In the $\operatorname{Sp}(4, R)$ model the sum has to be restricted to those states $|\gamma\rangle$ with $v=v_{0}$. With this restriction, eq. (69) is an approximation, since the sum no longer ranges over a complete set. Considerations involving sum rules ${ }^{21}$ ) and the detailed examination of special cases ${ }^{21}$ ) show that this is a very good approximation in practical calculations (at a large saving of computer time). In all final computations this approximation has therefore been made.

Matrix elements of the operators, $\operatorname{Tr}\left(Q^{\alpha}\right)$, can be evaluated with the use of the Wigner-Eckart theorem, eq. (55), with $\eta=\eta^{\mathrm{s}}=\kappa L M$. The $\mathrm{SU}(3)$ reduced matrix elements of the coupled operators $S_{\Gamma_{1} \Gamma_{2}}^{\Gamma_{\rho}}$ and $R_{\Gamma_{1} \Gamma_{2}: \Gamma_{3}}^{\Gamma_{12} \rho_{12} \overline{\sigma_{3}}}$ can be related to those for the generators $T^{\Gamma_{i}}$ by straightforward $\mathrm{SU}(3)$ recoupling tedchniques; [detailed derivations are given in ref. $\left.\left.{ }^{21}\right)\right]$

$$
\begin{aligned}
& \left\langle\Gamma^{\prime} v_{0} \gamma^{\prime}\left\|\left[T^{\Gamma_{1}} \times T^{\Gamma_{2}}\right]_{\bar{\rho}=1}^{\bar{\Gamma}}\right\| \Gamma v_{0} \gamma\right\rangle_{\rho} \\
& =\sum_{\tilde{\Gamma} \tilde{\gamma}} U\left(\Gamma_{1} \Gamma_{2} \Gamma^{\prime} \Gamma ; \bar{\Gamma} 1 \rho \tilde{\Gamma} 11\right)\left\langle\Gamma^{\prime} v_{0} \gamma^{\prime}\left\|T^{\Gamma_{1}}\right\| \tilde{\Gamma} v_{0} \tilde{\gamma}\right\rangle_{1}\left\langle\tilde{\Gamma} v_{0} \tilde{\gamma}\left\|T^{\Gamma_{2}}\right\| \Gamma v_{0} \gamma\right\rangle_{1},
\end{aligned}
$$




$$
\begin{aligned}
& \left\langle\Gamma^{\prime} v_{0} \gamma^{\prime}\left\|\left[\left[T^{\Gamma_{1}} \times T^{\Gamma_{2}}\right]_{\rho_{12}}^{\Gamma_{12}} \times T^{\Gamma_{3}}\right]_{\bar{\rho}}^{\bar{\Gamma}}\right\| \Gamma v_{0} \gamma\right\rangle_{\rho} \\
& =\sum_{\substack{\Gamma_{a} \gamma_{a} \\
\Gamma_{b} \gamma_{b} \tilde{\rho}}} U\left(\Gamma_{1} \Gamma_{2} \Gamma^{\prime} \Gamma_{a} ; \Gamma_{12} \rho_{12} \tilde{\rho} \Gamma_{b} 11\right) U\left(\Gamma_{12} \Gamma_{3} \Gamma^{\prime} \Gamma ; \bar{\rho} \rho \Gamma_{a} 1 \tilde{\rho}\right) \\
& \quad \times\left\langle\Gamma^{\prime} v_{0} \gamma^{\prime}\left\|T^{\Gamma_{1}}\right\| \Gamma_{b} v_{0} \gamma_{b}\right\rangle_{1}\left\langle\Gamma_{b} v_{0} \gamma_{b}\left\|T^{\Gamma_{2}}\right\| \Gamma_{a} v_{0} \gamma_{a}\right\rangle_{1}\left\langle\Gamma_{a} v_{0} \gamma_{a}\left\|T^{\Gamma_{3}}\right\| \Gamma v_{0} \gamma\right\rangle_{1} .
\end{aligned}
$$

The $U$-coefficients are SU(3) Racah coefficients ${ }^{18}$ ), with $\Gamma=(\lambda \mu)$.

\section{Applications}

With the technology developed in sect. 4, matrix elements of the quadrupole operator and the model hamiltonian, (44), can be evaluated in the $\operatorname{Sp}(4, R)$ basis symmetry-adapted to the physically relevant $\mathrm{SU}(3) \supset \mathrm{O}_{3}^{+}$subgroup chain. The new symplectic symmetry can be expected to be closest to an exact symmetry in those nuclei in which conventional shell-model calculations predict rotational bands dominated by a single $\mathrm{SU}(3)$ representation. Of these, the best candidates are found in nuclei in the $A=8-28$ mass range, with $A=4 n$, and ground-state wave functions dominated by states of $\left[4^{n}\right]$ space symmetry and $S=0=T$. The ground-state rotational bands in ${ }^{24} \mathrm{Mg}$ and ${ }^{20} \mathrm{Ne}$, and the rotational band built on the $6.05 \mathrm{MeV}$ $0^{+}$state in ${ }^{16} \mathrm{O}$ all show observed E2 rates in the $20-30 \mathrm{~W}$.u. range. Shell-model calculations indicate that these rotational bands can be expected to be dominated by states of the symplectic bands $\left\{\lambda_{0} \mu_{0} v_{0}\right\}=\{844\}$ for ${ }^{24} \mathrm{Mg},\{804\}$ for ${ }^{20} \mathrm{Ne}$, and $\{840\}$ for ${ }^{16} \mathrm{O}(6.05 \mathrm{MeV}$ band). For the latter the symplectic band head is a $4 \mathrm{p}-4 \mathrm{~h}$ state. Our aim is not to carry out a detailed spectroscopic study but to focus attention on the quadrupole collectivity in these bands. For an accurate fit of excitation energies and other nuclear properties the small admixtures of lower symmetries may be important. In ${ }^{24} \mathrm{Mg}$, e.g., conventional shell-model calculations ${ }^{27,28}$ ) predict a high percentage of $\operatorname{SU}(3)(\lambda \mu)=(84)$ symmetry. These shell-model calculations also predict smaller components with $(\lambda \mu)=(46),(08),(62), \ldots$ in the highest space symmetry $\left[4^{6}\right]$, and $(\lambda \mu)=(65), \ldots$ with lower space symmetry $\left[4^{5} 31\right]$. In the symplectic shell model each one of these can be expected to carry its own symplectic superstructure with built-in core excitations. A detailed description of the ${ }^{24} \mathrm{Mg}$ ground-state rotational band can thus be expected to involve a dominant $\left\{\lambda_{0} \mu_{0} v_{0}\right\}=$ $\{844\}$ symplectic component with small admixtures of $\{464\},\{084\},\{624\}, \ldots,\{654\}$, ... bands. However, our aim is to investigate the nature and the extent of the core excitations with are required to account for the observed quadrupole collectivity in such a band, using only the bare proton charge. For this purpose the idealized pure $\left\{\lambda_{0} \mu_{0} v_{0}\right\}=\{844\}$ model is sufficient. The quadrupole operator can not connect states with different $\left\{\lambda_{0} \mu_{0} v_{0}\right\}$. Admixtures with different $\left\{\lambda_{0} \mu_{0} \nu_{0}\right\}$ thus contribute incoherently to the $\mathrm{E} 2$ rates. In addition, the intrinsic quadrupole moments ${ }^{7}$ ) are proportional to $\left(2 \lambda_{0}+\mu_{0}\right)$. Admixtures with $\left\{\lambda_{0} \mu_{0} \nu_{0}\right\}$ values less than the dominant 
TABLE 5

$B\left(\mathrm{E} 2 ; K_{\mathrm{i}} L_{\mathrm{i}} \rightarrow K_{\mathrm{f}} L_{\mathrm{f}}\right)$ values for the $(8,4)$ ground-state band in ${ }^{24} \mathrm{Mg}^{\dagger}$

\begin{tabular}{|c|c|c|c|c|c|c|c|}
\hline \multirow[t]{2}{*}{$K_{i}$} & \multirow[t]{2}{*}{$L_{\mathrm{i}}$} & \multirow[t]{2}{*}{$K_{f}$} & \multirow[t]{2}{*}{$L_{\mathrm{f}}$} & \multirow[t]{2}{*}{ SU(3) } & \multicolumn{2}{|c|}{$\left.10 h \omega^{a}\right)$} & \multirow[t]{2}{*}{ Exp. $\left.{ }^{6}\right)$} \\
\hline & & & & & 19 & II $\left.{ }^{d}\right)$ & \\
\hline 0 & 2 & 0 & 0 & 6.9 & 26.3 & 20.5 & $20.5 \pm 6$ \\
\hline 0 & 4 & 0 & 2 & 9.5 & 36.5 & 28.0 & $23 \pm 4$ \\
\hline 0 & 6 & 0 & 4 & 9.7 & 39.3 & 30.4 & $34 \pm+10$ \\
\hline 0 & 8 & 0 & 6 & 9.0 & 39.6 & 29.4 & $16 \pm_{6}^{25}$ \\
\hline 0 & 10 & 0 & 8 & 7.2 & 36.1 & 25.0 & \\
\hline 2 & 4 & 2 & 2 & 3.6 & 12.2 & 8.2 & $16 \pm 3$ \\
\hline 2 & 5 & 2 & 3 & 5.7 & 21.1 & 16.1 & $31 \pm 5$ \\
\hline 2 & 6 & 2 & 4 & 5.6 & 18.2 & 12.8 & \\
\hline 2 & 7 & 2 & 5 & 6.7 & 26.1 & 19.6 & $23 \pm \pm_{8}^{23}$ \\
\hline 2 & 8 & 2 & 6 & 4.3 & 16.9 & 7.6 & $>3$ \\
\hline 2 & 9 & 2 & 7 & 5.7 & 25.6 & 17.4 & \\
\hline 2 & 2 & 0 & 0 & 2.5 & 4.6 & 2.4 & $1.4 \pm 0.3$ \\
\hline 2 & 4 & 0 & 2 & 1.3 & 0.5 & 0.1 & $1.0 \pm 0.2$ \\
\hline 2 & 6 & 0 & 4 & 0.8 & $<0.1$ & 0.3 & $0.8 \pm_{0.3}^{0.08}$ \\
\hline 2 & 8 & 0 & 6 & 0.4 & $<0.1$ & $<0.1$ & \\
\hline 0 & 10 & 2 & 8 & 2.0 & $<0.1$ & $<0.1$ & \\
\hline
\end{tabular}

${ }^{+} B$ (E2) strengths are given in Weisskopf units, where $1 \mathrm{~W} . \mathrm{u} .=4.112 e^{2} \cdot \mathrm{fm}^{4}$.

a) $h \omega=12.6 \mathrm{MeV}$.

b) $\operatorname{Ref}{ }^{31}$ ).

) Case I: $A_{2}=-0.10, A_{3}=-0.00065, A_{4}=0.000006$

d) Case II: $A_{2}=-0.06, A_{3}=-0.00025, A_{4}=0.000005$.

$\{844\}$ can thus be expected to decrease the predicted E2 rates. To get a realistic measure of the amount of core excitation needed to account for observed E2 rates the idealized pure $\left\{\lambda_{0} \mu_{0} v_{0}\right\}=\{844\}$ model can be used. If this model fits the observed E2 rates, the components with smaller symplectic quantum numbers $\left\{\lambda_{0}^{\prime} \mu_{0}^{\prime} v_{0}^{\prime}\right\}$ in a more detailed model can only be expected to decrease the predicted E2 rates. For this reason, two types of fits have generally been carried out, one in which the observed $2_{1}^{+} \rightarrow 0_{1}^{+} B(E 2)$ value is fitted exactly by the idealized $\left\{\lambda_{0} \mu_{0} v_{0}\right\}$ symplectic model, and another in which this model gives a slight overprediction for this $B(E 2)$ value. Results for the ground-state band in ${ }^{24} \mathrm{Mg}$ are shown in table 5 and fig. 3. The three parameters $A_{2}, A_{3}, A_{4}$ of the model hamiltonian, (44), were adjusted to give an approximate fit to the excitation energies and the desired fit to the observed $2_{1}^{+} \rightarrow 0_{1}^{+}$ $B(E 2)$ values. The energy diagonalizations were carried out in the restricted $\operatorname{Sp}(4, \mathbf{R})$ model basis, truncated to include core excitations with excitation energy $\leqq N_{\max } h \omega$. Usually $N_{\max }=10$ was sufficient to insure convergence. Although quite different parameter sets could give comparable fits to the energy spectra, two different parameter sets which gave identical $B(E 2)$ fits always led to wave functions of comparable 


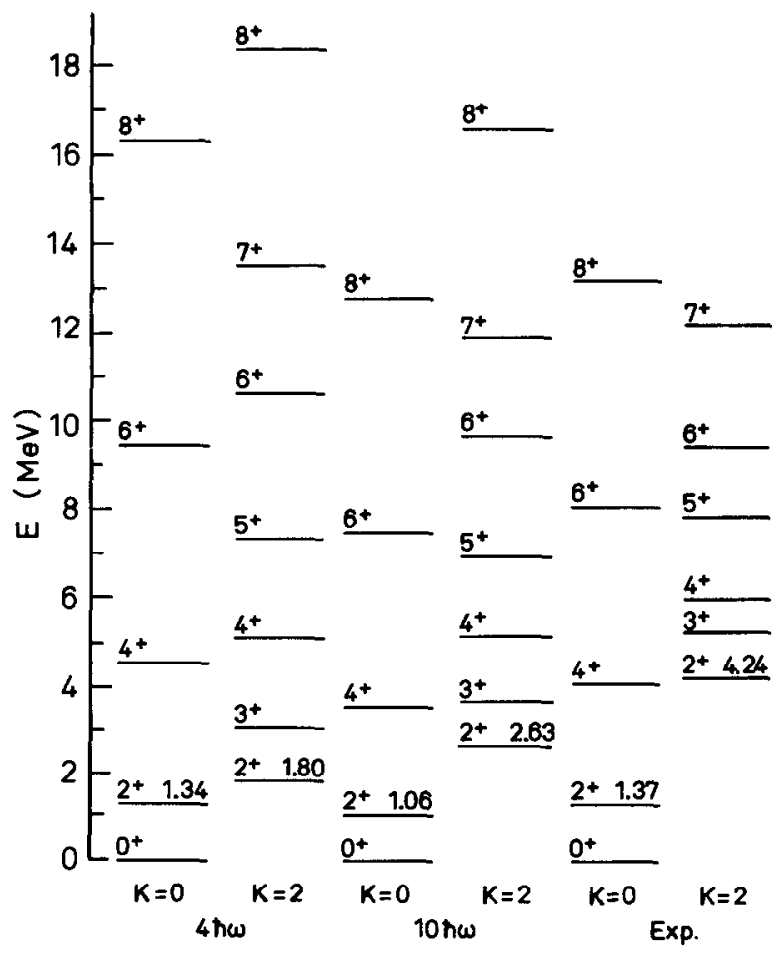

Fig. 3. Spectra of $K=0$ and $K=2$ rotational bands in ${ }^{24} \mathrm{Mg}$ built on the ground state, $(8,4)$ symplectic band head.

character, so that the nature and extent of the core excitations needed to fit observed $\mathrm{E} 2$ rates do not seem to be dependent on the precise values of the model parameters $A_{2}, A_{3}, A_{4}$. In table 5 the predicted $B(\mathrm{E} 2)$ values for two calculations with $N_{\max }=10$ are compared with the experimental $B(\mathrm{E} 2)$ values for ${ }^{24} \mathrm{Mg}$. The column marked $\mathrm{SU}(3)$ gives the $B(\mathrm{E} 2)$ values predicted by the $\mathrm{SU}(3)$ shell model, $\left(N_{\max }=0\right)$. For E2 transitions between $T=0$ states, only the isoscalar part of the E2 operator can make a contribution, so that

$$
\mathscr{M}(\mathrm{E} 2 ; \mu)=\frac{1}{2} e Q_{\mu}^{(2)} \quad \text { for } T_{\mathrm{i}}=T_{\mathrm{f}}=0 .
$$

We stress again the absence of any effective charges in this equation; $e$ is the bare proton charge. The oscillator length parameter for ${ }^{24} \mathrm{Mg}$ was chosen as $(\hbar / 2 m \omega)^{\frac{1}{2}}=$ $1.28 \mathrm{fm}$, corresponding to $\left.{ }^{30}\right)$ an $\hbar \omega=\left(45 / A^{\frac{1}{3}}-25 / A^{\frac{2}{3}}\right)=12.6 \mathrm{MeV}$. Table 5 shows the parameters $A_{2}, A_{3}$ and $A_{4}$ for the two calculations, in MeV. Fig. 3 shows the energy spectra for the set 1 for two different truncations, with $N_{\max }=4$ and 10 . Although the full calculation ( $N_{\max }=10$ ) pushes the $K=2$ band up relative to the $K=0$ band, these calculations share a feature of many conventional sd-shell model calculations ${ }^{27-29}$ ): the predicted $K=2$ band head lies too low in energy. However, we stress again the fact that the idealized pure $\left\{\lambda_{0} \mu_{0} v_{0}\right\}$ model can not be expected 
TABLE 6

Wave functions of the ground state $(8,4)$ band in the ${ }^{24} \mathrm{Mg}, 10 \mathrm{h \omega}$ calculation ${ }^{\dagger}$

\begin{tabular}{|c|c|c|c|c|c|c|c|c|}
\hline$(\lambda \mu)$ & $\kappa$ & $\gamma$ & $0_{1}^{+}$ & $2_{1}^{+}$ & $4_{1}^{+}$ & $6_{1}^{+}$ & $8_{1}^{+}$ & $10_{1}^{+}$ \\
\hline$(8,4)$ & 1 & 1 & \multirow[t]{2}{*}{-0.562} & 0.558 & 0.522 & 0.477 & 0.469 & 0.503 \\
\hline$(8,4)$ & 2 & 1 & & 0.050 & 0.185 & 0.260 & 0.237 & -0.004 \\
\hline$(10,4)$ & 1 & 1 & \multirow[t]{3}{*}{0.587} & -0.582 & -0.542 & -0.489 & -0.474 & -0.482 \\
\hline$(10,4)$ & 2 & 1 & & -0.057 & -0.212 & -0.310 & -0.318 & -0.277 \\
\hline$(8,5)$ & 1 & 1 & & 0.047 & 0.063 & 0.061 & 0.067 & 0.105 \\
\hline$(12,4)$ & 1 & 1 & \multirow[t]{3}{*}{-0.471} & 0.466 & 0.433 & 0.389 & 0.374 & 0.381 \\
\hline$(12,4)$ & 2 & 1 & & 0.048 & 0.179 & 0.266 & 0.286 & 0.274 \\
\hline$(10,5)$ & 1 & 1 & & -0.062 & -0.081 & -0.074 & -0.073 & -0.083 \\
\hline$(14,4)$ & 1 & 1 & \multirow[t]{3}{*}{0.293} & -0.289 & -0.270 & -0.244 & -0.237 & -0.245 \\
\hline$(14,4)$ & 2 & 1 & & -0.031 & -0.115 & -0.175 & -0.195 & -0.198 \\
\hline$(12,5)$ & 1 & 1 & & 0.048 & 0.062 & 0.056 & 0.053 & 0.057 \\
\hline$(16,4)$ & 1 & 1 & \multirow[t]{3}{*}{-0.133} & 0.133 & 0.125 & 0.115 & 0.115 & 0.123 \\
\hline$(16,4)$ & 2 & 1 & & 0.014 & 0.054 & 0.085 & 0.098 & 0.106 \\
\hline$(14,5)$ & 1 & 1 & & -0.026 & -0.034 & -0.030 & -0.029 & -0.030 \\
\hline$(18,4)$ & 1 & 1 & \multirow[t]{3}{*}{0.034} & -0.035 & -0.034 & -0.033 & -0.035 & -0.040 \\
\hline$(18,4)$ & 2 & 1 & & -0.004 & -0.015 & -0.024 & -0.031 & -0.036 \\
\hline$(16,5)$ & 1 & 1 & & 0.008 & 0.011 & 0.010 & 0.010 & 0.011 \\
\hline \multicolumn{3}{|c|}{$\left.\sum K \ldots\left|L^{\pi}\right\rangle\right|^{2}$} & 0.99 & 0.99 & 0.99 & 0.98 & 0.96 & 0.93 \\
\hline
\end{tabular}

${ }^{+} A_{2}=-0.06, A_{3}=-0.00025, A_{4}=0.000005$.

to give accurate predictions for excitation energies. Our aim is to examine the nature of the core excitations. These are shown in table 6 which gives the amplitudes of the most important components of the wave functions of the ground state rotational band. We choose the parameter set II which fits the $2_{1}^{+} \rightarrow 0_{1}^{+} B(E 2)$ value exactly. [In ${ }^{24} \mathrm{Mg}$ the admixtures of smaller $\left\{\lambda_{0} \mu_{0} v_{0}\right\}$ in a more detailed symplectic-symmetry breaking model can be expected to have relatively large values of $\left(2 \lambda_{0}+\mu_{0}\right)$; and in ${ }^{24} \mathrm{Mg}$ an admixture of $\left\{\lambda_{0} \mu_{0} v_{0}\right\}=\{14,2,4\}$, a symplectic band based on the $\left(\mathrm{s}^{4} \mathrm{p}^{12} \mathrm{sd}^{6} \mathrm{pf}^{2}\right) 2 \mathrm{p}-2 \mathrm{~h}$ shell-model configuration may, e.g., compensate for the lowering of the $B(E 2)$ values due to admixtures of smaller $\left\{\lambda_{0} \mu_{0} v_{0}\right\}$.]

We note the following about the nature of these wave functions:

(i) To fit the observed $2_{1}^{+} \rightarrow 0_{1}^{+}$E2 rate exactly, large core excitations are required. States with $2 \hbar \omega$ excitation energy and $(\lambda \mu)=(10,4)$, and $4 \hbar \omega$ excitation energy and $(\lambda \mu)=(12,4)$ have amplitudes comparable to that of the "shell-model state" with $(\lambda \mu)=(84)$. The admixtures of core excited states are too large to be treated by conventional perturbation theory.

(ii) The truncation of the $\mathrm{Sp}(4, \mathrm{R})$ model space with excitation energies $\leqq N_{\max } \hbar \omega$ converges relatively rapidly beyond $N_{\max }=6$. The components with $N_{\max }=10$ in table 6 contribute less than $0.15 \%$ of the intensity of the total wave functions.

(iii) Despite the large amount of core excitation, the similarities in the wave functions for the $0_{1}^{+}, 2_{1}^{+}, 4_{1}^{+}, \ldots, 10_{1}^{+}$states are apparent. These clearly form a band of states, the symplectic rotational band $\{844\}$. (States with $\kappa=1$ are pure Elliott 
$K=0$ states, those with $\kappa=2$ predominantly $K=2$ states ${ }^{18}$ ). This lowest rotational band is predominantly $K=0$, with non-negligible $K=2$ admixtures in the higher members of the band).

(iv) The major part of the core excitations is carried by a few very simple states. Table 6 lists only the dominant components of the wave functions. For $N_{\max }=10$, the dimension of the $\operatorname{Sp}(4, \mathrm{R})$ model space ranges from 33 for $0^{+}$states to 186 for $8^{+}$states; yet $99 \%$ of the intensity of the $0_{1}^{+}$state comes from $6 \mathrm{Sp}(4, \mathrm{R})$ states, and $96 \%$ of the intensity of the $8_{1}^{+}$state from $17 \mathrm{Sp}(4, \mathrm{R})$ states. Comparison with fig. 2 shows that all of the $(\lambda \mu)$ listed in table 6 fall on two vertical lines of fig. 2, the $z$ or $\left(M_{A}\right)$ axis, and the nearest line which parallels the $z$ axis. Clearly states along the $z$-ladder are by far the most important, carrying more than $94 \%$ of the intensity of all states. The $S p(4, R)$ model space could have been truncated further to states along the $z$-ladder plus those with 1 or at most 2 excitation quanta in the $x$-direction. This result certainly supports the original truncation from $D^{+}\left(\left\{\lambda_{0} \mu_{0} v_{0}\right\}\right)$ to $D^{++}(\bar{\Lambda} \bar{\Sigma})$, since $y$-excitations are even less important, as also observed directly for ${ }^{20} \mathrm{Ne}$ by Rosensteel and Rowe ${ }^{17}$ ). Even more, the $\mathrm{Sp}(2, R)_{z}$ symmetry of Arickx is a relatively good symmetry for this band despite the fact that $\mu_{0}=4$ is relatively large.

Very similar conclusions can be reached from calculations ${ }^{21}$ ) for the $\{804\}$ band in ${ }^{20} \mathrm{Ne}$. In this regard there is some qualitative difference with the results for ${ }^{20} \mathrm{Ne}$ of ref. ${ }^{17}$ ) which seems to indicate that the core excitations in ${ }^{20} \mathrm{Ne}$ can be treated by perturbation theory. It should be pointed out, however, that the ${ }^{20} \mathrm{Ne}$ wave functions exhibited in ref. ${ }^{17}$ ) account for only $70-80 \%$ of the observed E2 rates. In view of the expected admixtures of smaller $\left(\lambda_{0} \mu_{0}\right)$ shell-model components $\left.{ }^{32}\right)$, a $100 \%$ or a slight overprediction is needed for the idealized pure $\{804\}$ model to gain a realistic view of the actual quadrupole core excitations. In this regard our ${ }^{20} \mathrm{Ne}$ results agree with those of Arickx et al. ${ }^{22}$ ) for ${ }^{8} \mathrm{Be}$ for the $\infty$-dimensional $\operatorname{Sp}(2, \mathbf{R})_{z}$ symplectic model, which again shows that states with core excitation energies of $0 \hbar \omega, 2 \hbar \omega$ and $4 \hbar \omega$ are of comparable importance.

Results for the rotational band based on the $6.05 \mathrm{MeV} 0^{+}$state in ${ }^{16} \mathrm{O}$ are very similar to those for the ${ }^{24} \mathrm{Mg}$ ground-state rotational band. The symplectic band $\left\{\lambda_{0} \mu_{0} v_{0}\right\}=\{840\}$ based on the dominant $4 \mathrm{p}-4 \mathrm{~h}$ shell-model state consists of the irrep $D^{++}\left(\bar{\Lambda}=\frac{39}{4}, \bar{\Sigma}=\frac{23}{4}\right)$ in the $\mathrm{Sp}(4, \mathbf{R})$ model subspace. (In contrast, the appropriate $\mathrm{Sp}(4, \mathrm{R})$ space for the $\{844\}$ symplectic band of ${ }^{24} \mathrm{Mg}$ was $D^{++}\left(\bar{\Lambda}=\frac{55}{4}\right.$, $\left.\bar{\Sigma}=\frac{39}{4}\right)$.) A calculation which fits the observed $\left.{ }^{33}\right) 2^{+}(6.92 \mathrm{MeV}) \rightarrow 0^{+}(6.05 \mathrm{MeV})$ $B(\mathrm{E} 2)$ value of $17 \mathrm{~W}$.u. leads to wave functions of structure almost identical to those exhibited in table 6. In this calculation, $A_{2}=-0.08, A_{3}=-0.0009, A_{4}=0.000006$ $\mathrm{MeV}$. The $2_{1}^{+}$state, e.g., has the structure

$$
\begin{aligned}
\left|2_{1}^{+}\right\rangle=0.55|(8,4)\rangle-0.58|(10,4)\rangle+0.47|(12,4)\rangle \\
-0.29|(14,4)\rangle+0.13|(16,4)\rangle-0.03|(18,4)\rangle+\ldots .
\end{aligned}
$$

Since the $6.05 \mathrm{MeV}$ rotational band in ${ }^{16} \mathrm{O}$ is expected ${ }^{34}$ ) to have components based 
TABLE 7

Wave functions of the $0 \mathrm{p}-0 \mathrm{~h},(00)$ band in the ${ }^{16} \mathrm{O}, 10 \hbar \omega$ calculation ${ }^{\dagger}$

\begin{tabular}{|c|c|c|c|c|c|c|c|}
\hline$(\lambda \mu)$ & $\kappa$ & $0_{1}^{+}$ & $2_{1}^{+}$ & $4_{1}^{+}$ & $6_{1}^{+}$ & $8_{1}^{+}$ & $10_{1}^{+}$ \\
\hline$(0,0)$ & I & -0.957 & & & & & \\
\hline$(2,0)$ & 1 & 0.150 & -0.918 & & & & \\
\hline$(4,0)$ & 1 & -0.187 & -0.086 & -0.869 & & & \\
\hline$(0,2)$ & 1 & 0.153 & -0.171 & & & & \\
\hline$(6,0)$ & 1 & 0.011 & -0.201 & -0.259 & 0.864 & & \\
\hline$(2,2)$ & 1 & -0.005 & -0.225 & -0.203 & & & \\
\hline$(2,2)$ & 2 & & 0.129 & & & & \\
\hline$(8,0)$ & 1 & -0.026 & -0.046 & -0.217 & 0.315 & -0.915 & \\
\hline$(4,2)$ & 1 & -0.029 & -0.046 & -0.233 & 0.181 & & \\
\hline$(4,2)$ & 2 & & -0.019 & 0.132 & & & \\
\hline$(0,4)$ & 1 & -0.031 & 0.033 & -0.060 & & & \\
\hline$(10,0)$ & 1 & $<0.001$ & -0.036 & -0.074 & 0.205 & -0.334 & 1.0 \\
\hline$(6,2)$ & 1 & -0.001 & -0.046 & -0.078 & 0.232 & -0.225 & \\
\hline$(6,2)$ & 2 & & 0.015 & -0.002 & -0.151 & & \\
\hline$(2,4)$ & 1 & $<0.001$ & -0.008 & 0.019 & 0.057 & & \\
\hline$(2,4)$ & 2 & & -0.057 & 0.049 & & & \\
\hline \multicolumn{2}{|c|}{$\left.\sum|\ldots| L^{\pi}\right\rangle\left.\right|^{2}$} & 1.0 & 1.0 & 1.0 & 1.0 & 1.0 & 1.0 \\
\hline
\end{tabular}

TABLE 8

Wave functions of the $2 \mathrm{p}-2 \mathrm{~h},(42)$ band in the ${ }^{16} \mathrm{O}, 10 \hbar \omega$ calculation ${ }^{\dagger}$

\begin{tabular}{|c|c|c|c|c|c|c|c|c|}
\hline$(\lambda \mu)$ & $\kappa$ & $\gamma$ & $0_{1}^{+}$ & $2_{1}^{+}$ & $4_{1}^{+}$ & $6_{1}^{+}$ & $8_{1}^{+}$ & $10_{1}^{+}$ \\
\hline$(4,2)$ & 1 & 1 & \multirow[t]{2}{*}{-0.873} & 0.879 & 0.844 & \multirow[t]{2}{*}{0.936} & & \\
\hline$(4,2)$ & 2 & 1 & & 0.079 & 0.322 & & & \\
\hline$(6,2)$ & 1 & 1 & \multirow[t]{3}{*}{-0.370} & 0.354 & 0.292 & 0.219 & \multirow[t]{3}{*}{0.810} & \\
\hline$(6,2)$ & 2 & 1 & & 0.041 & 0.154 & 0.167 & & \\
\hline$(4,3)$ & 1 & 1 & & -0.028 & -0.040 & -0.052 & & \\
\hline$(8,2)$ & 1 & 1 & \multirow[t]{3}{*}{-0.240} & 0.223 & 0.171 & 0.109 & 0.384 & 0.724 \\
\hline$(8,2)$ & 2 & 1 & & 0.029 & 0.099 & -0.099 & 0.235 & \\
\hline$(6,3)$ & 1 & 1 & & -0.053 & -0.055 & -0.035 & -0.057 & \\
\hline$(10,2)$ & 1 & 1 & \multirow[t]{3}{*}{-0.132} & 0.121 & 0.089 & 0.053 & 0.235 & 0.482 \\
\hline$(10,2)$ & 2 & 1 & & 0.016 & 0.052 & -0.049 & 0.172 & 0.251 \\
\hline$(8,3)$ & 1 & 1 & & -0.025 & -0.024 & -0.013 & -0.046 & -0.054 \\
\hline$(12,2)$ & 1 & 1 & \multirow[t]{3}{*}{-0.072} & 0.065 & 0.047 & 0.123 & 0.132 & 0.305 \\
\hline$(12,2)$ & 2 & 1 & & 0.009 & 0.027 & 0.025 & 0.102 & 0.195 \\
\hline$(10,3)$ & 1 & 1 & & -0.015 & -0.013 & -0.007 & -0.025 & -0.047 \\
\hline$(14,2)$ & 1 & 1 & \multirow[t]{3}{*}{-0.032} & 0.029 & 0.020 & 0.015 & 0.062 & 0.152 \\
\hline$(14,2)$ & 2 & 1 & & 0.004 & 0.012 & 0.001 & 0.049 & 0.104 \\
\hline$(12,3)$ & 1 & 1 & & -0.006 & -0.006 & -0.003 & -0.013 & -0.027 \\
\hline \multicolumn{3}{|c|}{$\sum\left|\left\langle\ldots \mid L^{\pi}\right\rangle\right|^{2}$} & 0.98 & 0.98 & 0.98 & 0.98 & 0.98 & 0.98 \\
\hline
\end{tabular}

${ }^{\dagger} A_{2}=-0.06, A_{3}=-0.00045, A_{4}=0.000003$. 
on $0 \mathrm{p}-0 \mathrm{~h}$ and $2 \mathrm{p}-2 \mathrm{~h}$ shell-model states with low $\lambda_{0}, \mu_{0}$ values, the results of a second calculation with a predicted $2^{+}(6.92 \mathrm{MeV}) \rightarrow 0^{+}(6.05 \mathrm{MeV}) B(\mathrm{E} 2)$ value of $25.7 \mathrm{~W} . \mathrm{u}$. may also be of interest. In this calculation, $A_{2}=-0.09, A_{3}=-0.00065, A_{4}=$ $0.000006 \mathrm{MeV}$; and now

$$
\begin{aligned}
\left|2_{1}^{+}\right\rangle=0.17|(8,4)\rangle-0.35|(10,4)\rangle+0.50|(12,4)\rangle-0.55|(14,4)\rangle \\
+0.46|(16,4)\rangle-0.26|(18,4)\rangle+\ldots .
\end{aligned}
$$

To see that the large core excitations of eqs. (73) and (74) are related to the large values of the quantum numbers $\lambda_{0}, \mu_{0}$, table 7 shows the amplitudes for the most important core excitations in the $\left\{\lambda_{0} \mu_{0} v_{0}\right\}=\{004\}$ symplectic band based on the $0 \mathrm{p}-0 \mathrm{~h}$ state of ${ }^{16} \mathrm{O}$ with $\left(\lambda_{0} \mu_{0}\right)=(00)$. For this case $\bar{A}=\bar{\Sigma}=\frac{23}{4}$. The parameters $A_{i}$ for this band were chosen to give an excitation energy of $21.5 \mathrm{MeV}$ for the $1 \mathrm{st}$ excited $2^{+}$state, corresponding to the position of the observed giant E2 resonance ${ }^{35}$ ). Note that the $A_{i}$ of table 7 are essentially identical to those used in connection with eq. (73); yet the (00) state carries $91 \%$ of the $0_{1}^{+}$intensity, and core excitations are weak. Finally, table 8 shows the wave functions for the $\{422\}$ band in ${ }^{16} \mathrm{O}$, with $\bar{\Lambda}=\frac{31}{4}, \bar{\Sigma}=\frac{23}{4}$ based on the most deformed $2 \mathrm{p}-2 \mathrm{~h}$ state with $\left(\lambda_{0} \mu_{0}\right)=(42)$.

\section{Conclusions}

The sample calculations presented in this investigation show that the $\operatorname{Sp}(4, R)$ shell model of collective motion strikes a practical compromise between the two extreme models, $S p(2, R)$ and $S p(6, R)$. This model avoids some of the computational complexities associated with the full $\mathrm{Sp}(6, \mathrm{R})$ symmetry; and yet core excitations with large values of both $\mathrm{SU}(3)$ quantum numbers $\lambda$ and $\mu$ are included in the model. This may be important in nuclei in which there is competition between prolate and oblate intrinsic shapes. In nuclei with large prolate deformations the calculations indicate that the $\operatorname{Sp}(4, \mathbf{R})$ model space can be truncated further to include only excitations along the $z$-ladder and states with only one or two excitation quanta in the $x$-direction, for which matrix elements can be written in simple analytic form.

The core excitations needed to account for quadrupole collective effects in strongly deformed nuclei are too large to be treated by perturbation theory, so that conventional shell-model calculations with effective charges may give an oversimplified picture of quadrupole collectivity. Despite the large core excitations, a definite symplectic band structure emerges for the experimentally observed rotational bands in light nuclei.

Large core excitations seem to be required mainly for symplectic bands $\left\{\lambda_{0} \mu_{0} v_{0}\right\}$ with large quantum numbers $\lambda_{0}, \mu_{0}$. Since these excitations have a very simple structure, it seems feasible to incorporate the symplectic core excitations into much more detailed shell-model calculations. Large symplectic core excitations may be required only for a few shell-model components with large $\lambda_{0}, \mu_{0}$ values; and for many of these the core excitation calculations can be simplified through use of the $\operatorname{Sp}(2, \mathbf{R})_{z}$ model or a truncation to a very simple subspace of the $\operatorname{Sp}(4, R)$ model. 


\section{References}

1) A. Bohr and B. R. Mottelson, Nuclear structure, Vol. II (Benjamin, Reading, Mass., 1975)

2) G. Rosensteel and D. J. Rowe, Phys. Rev. Lett. 38 (1977) 10

3) G. Rosensteel and D. J. Rowe, in Group theoretical methods in physics, ed. P. Kramer and A. Rieckers (Springer, Berlin, 1978)

4) H. Ui, Prog. Theor. Phys. 44 (1970) 153

5) O. L. Weaver, L. C. Biedenharn and R. Y. Cusson, Ann. of Phys. 77 (1973) 250

6) G. Rosensteel and D. J. Rowe, Ann. of Phys. 96 (1976) 1

7) J. P. Elliott, Proc. Roy. Soc. A245 (1958) 128; 562;

J. P. Elliott and M. Harvey, Proc. Roy. Soc. A272 (1963) 55;

M. Harvey, in Advances in nuclear physics, Vol. 1, ed. M. Baranger and E. Vogt (Plenum, New York, 1968)

8) B. G. Wybourne, Classical groups for physicists (Wiley, New York, 1974)

9) S. Goshen and H. J. Lipkin, Ann. of Phys. 6 (1959) 301

10) C. Quesne and M. Moshinsky, J. Math. Phys. 12 (1971) 1780

11) F. Arickx, Nucl. Phys. A268 (1976) 347

12) F. Arickx, P. van Leuven and M. Bouten, Nucl. Phys. A252 (1975) 416

13) R. Gilmore, Lie groups, Lie algebras and some of their applications (Wiley, New York, 1974)

14) A. O. Barut, in Lectures in theoretical physics, ed. W. E. Brittin, A. O. Barut and M. Guenin (Gordon and Breach, New York, 1967)

15) H. Ui, Ann. of Phys. 49 (1968) 69

16) G. Rosensteel, J. Math. Phys. 21 (1980) 924

17) G. Rosensteel and D. J. Rowe, to be published

18) J. P. Draayer and Y. Akiyama, J. Math. Phys. 14 (1973) 1904;

Y. Akiyama and J. P. Draayer, Comput. Phys. Commun. 5 (1973) 405

19) P. J. Ellis and E. Osnes, Rev. Mod. Phys. 49 (1977) 777

20) R. M. Thrall, Am. J. Math. 64 (1942) 371

21) D. R. Peterson, Ph.D. Thesis, University of Michigan, Ann Arbor, Michigan (1979)

22) F. Arickx, J. Broeckhove and E. Deumens, Nucl. Phys. A318 (1979) 269

23) S. Goshen and H. J. Lipkin, in Spectroscopic and group theoretical methods in physics, ed. F. Bloch (North-Holland, Amsterdam, 1968)

24) K. T. Hecht, Nucl. Phys. 62 (1965) 1

25) A. P. Stone, Proc. Cambridge Phil. Soc. 57 (1961) 460

26) K. T. Hecht, Nucl. Phys. 63 (1965) 177

27) Y. Akiyama et al., Nucl. Phys. A138 (1969) 273

28) H. Feldmeier, P. Manakos and T. Wolff, Z. Phys. 258 (1973) 81

29) D. Strottman, Phys. Lett. 39B (1972) 457;

R. R. Whitehead and A. Watt, Phys. Lett. 41B (1972) 7;

J. B. McGrory and B. H. Wildenthal, Phys. Lett. 34B (1971) 373

30) J. Blomquist and A. Molinari, Nucl. Phys. A106 (1968) 545;

G. F. Bertsch, The practitioner's shell model (North-Holland, Amsterdam, 1972)

31) D. Branford, A. C. McGough and I. F. Wright, Nucl. Phys. A241 (1975) 349;

M. P. Fewell, S. Hinds, D. C. Kean and T. H. Zabel, Nucl. Phys. A319 (1979) 214;

S. F. Biagi, W. R. Phillips and A. R. Barnett, Nucl. Phys. A242 (1975) 160;

D. Schwalm, E. K. Warburton and J. W. Olness, Nucl. Phys. A293 (1977) 425

32) T. Tomoda and A. Arima, Nucl. Phys. A303 (1978) 217

33) F. Ajzenberg-Selove, Nucl. Phys. A116 (1971) 1

34) G. E. Brown and A. M. Green, Nucl. Phys. 75 (1966) 401

35) K. T. Knöpfle, G. J. Wagner, II. Breuer, M. Rogge and C. Mayer-Böricke, Phys. Rev. Lett. 35 (1975) 779 